\title{
ANÁLISE DE CONFIABILIDADE EM SISTEMAS REPARÁVEIS COMPLEXOS
}

\author{
Marco César dos Santos Barbosa
}

\begin{abstract}
Dissertação Apresentada ao Instituto de MATEMÁtica E Estatística da UNIVERsidade de São paulo para Obtençāo do Título de Mestre em Estatística
\end{abstract}

Área de Concentração: Estatística

Orientador: Prof. Dr. Wagner de Souza Borges 


\title{
ANÁLISE DE CONFIABILIDADE EM SISTEMAS REPARÁVEIS COMPLEXOS
}

\author{
Este exemplar corresponde à redação \\ final da dissertação devidamente \\ corrigida e defendida por Marco \\ César dos Santos Barbosa, e apro- \\ vada pela comissão julgadora.
}

São Paulo, agosto de 2005.

Comissão julgadora:

- Prof. Dr. Wagner de Souza Borges (Orientador) - IME-USP

- Prof. Dr. Carlos Alberto de Bragança Pereira - IME-USP

- Prof. Dra. Linda Lee Ho - EP-USP 


\section{Agradecimentos}

Gostaria de agradecer primeiro a Deus por todas as benções recebidas em minha vida pessoal, profissional e acadêmica.

Aos meus pais, Achelon e Virtuosa, por sua paciência, amor e dedicação e por patrocinarem todas as fases de meus estudos e me incentivar nos momentos mais difíceis.

Ao meu orientador e amigo, Prof. Dr. Wagner de Souza Borges, por compartilhar comigo seu tema preferido de pesquisa e também todo o seu conhecimento referente a este, sendo um interlocutor disposto a oferecer estímulos e também calar as dúvidas. Por sua amizade, paciência, dedicação e principalmnte pela coragem e ousadia de trabalhar no desenvolvimento de novas idéias e conceitos.

Ao amigo Jorge Gonella no qual me inspiro profissionalmente, por sua seriedade, competência, caráter, dignidade e por ter me apoiado e incentivado nas minhas pesquisas.

Aos docentes do departamento de estatística da Universidade de São Paulo e a todos aqueles que de uma maneira direta ou indireta contribuiram na minha formação profissional e acadêmica, despertando em mim o interesse pela arte de trabalhar com números. 


\section{Resumo}

Os modelos mais comuns utilizados para descrever o fluxo de falhas de sistemas reparáveis são os processos de Poisson homogêneo e não-homogêneo, e o Processo de renovação. Este trabalho tem como objetivo descrever o método de análise da confiabilidade de sitemas reparáveis complexos, utilizando o modelo misto, que incorpora, simultaneamente, os padrões poissoniano e de renovação na modelagem da função de intensidade completa. A intenção é mostrar a flexibilidade e o poder deste modelo na análise de dados de falhas recorrentes.

\section{Abstratct}

The most commonly used models for the failure process of a repairable system are the homogeneous and nonhomogeneous Poisson processes, and the renewal processes. The objective of this dissertation is to describe a reliability analysis method for complex repairable systems, based on the mixed model, which incorporates both trend (Poison) and renewal behavior into the complete intensity function. The propose is to show the flexibility and power of the mixed model in the analysis of recurrent failure data. 


\section{Introdução}

Uma das dimensões mais importantes da qualidade de um produto é a confiabilidade. Para atingir objetivos estratégicos e comerciais tais como melhorar sua posição competitiva e seu market share, além de encantar e cativar os consumidores de seus produtos, várias empresas, especialmente as de alta tecnologia, tem adotado programas de qualidade centrados na engenharia da confiabilidade.

A disciplina de engenharia da confiabilidade foi introduzida nas décadas de 40 e 50 pelas autoridades militares norte americanas. Inicialmente, essa disciplina foi utilizada pelas indústrias bélica e aeronáutica, mas, a partir dos anos 70 foi também aplicada intensamente pela indústria nuclear norte americana para avaliar a segurança das instalações por ela projetadas e construídas.

Com a evolução das técnicas de gestão da qualidade e o aumento do nível de exigência dos consumidores de produtos industrializados, a engenharia da confiabilidade passou a ser utilizada também em ambientes de nível tecnológico mais baixo. Neste contexto, o foco dos estudos concentrou-se na avaliação do tempo de vida de componentes. Com a popularização dessa disciplina, inúmeras obras foram elaboradas e editadas para auxiliar, principalmente, os engenheiros da qualidade, confiabilidade e desenvolvimento de produtos, no planejamento e análise de testes de componentes. Várias obras foram dedicadas também à engenharia da confiabilidade de sistemas complexos, isto é, conjuntos de componentes que funcionam de forma simultânea e interagente com um objetivo comum. O termo sistema complexo 
viii

é adotado aqui e em outros trabalhos para designar sistemas que podem ser subdivididos em vários subsistemas como, por exemplo, o sistema veículo, que é geralmente subdividido em subsistemas tais como motor, transmissão, suspensão, chassi e outros.

Nos últimos anos, entretanto, várias publicações tem voltado o seu foco para a metodologia científica destinada a tratar a confiabilidade de sistemas reparáveis, isto é, sistemas que após a ocorrência de uma falha podem ter a sua funcionalidade recuperada, por algum tipo de ação restauradora ou de manutenção (reparo ou substituição de componentes).

Tendo em vista a importância dessa metodologia para as indústrias aeronáutica, mecânica, automobilística e eletro-eletrônica, e aproveitando a experiência obtida com a sua utilização em estudos de confiabilidade desenvolvidos para a indústria automotiva, este trabalho tem como objetivo apresentar essa metodologia de forma didática, descrevendo e discutindo a utilização de modelos probabilísticos típicos da análise da confiabilidade de sistemas reparáveis.

Para tanto, noções básicas de processos pontuais e conceitos fundamentais para o desenvolvimento deste trabalho são introduzidas no Capítulo 1. Nesse capítulo, introduz-se ainda os modelos mais utilizados e importantes. Especificamente, os processos pontuais de Poisson e de Renovação.

O Capítulo 2 é dedicado à inferência para três famílias de modelos: as famílias de processos pontuais de Poisson não-homogêneos com intensidade do tipo power-law e log-linear, e a família de modelos mistos, que incorpora tanto os processos pontuais de Poisson quanto os de renovação. Essa flexibilidade torna a família de modelos mistos extremamente interessante na análise da confiabilidade de sistemas, na medida em que possibilita o tratamento unificado de modelos que até recentemente eram tratados isoladamente.

No Capítulo 3, apresenta-se, algumas aplicações dos modelos discutidos no Capítulo 2. 
E finalmente no Apendice, é disponibilizado um dos programas desenvolvidos em S-Plus para a aplicação do modelo misto em um conjunto de dados (única unidade amostral). 


\section{Índice}

Lista de Figuras $\quad$ xiii

1 Processos Pontuais 1

1.1 Processos Pontuais Simples . . . . . . . . . . . . 6

1.2 Outros Processos Pontuais . . . . . . . . . . . . . 10

2 Inferência Estatística dos Modelos $\quad 13$

2.1 Modelo Power-Law . . . . . . . . . . . . . . . . . . . . . . 13

2.1.1 Dados com Censura Tipo I . . . . . . . . . . . . . . . 14

2.1.2 Dados com Censura Tipo II . . . . . . . . . . . . 17

2.2 Modelo Log-Linear . . . . . . . . . . . . . . . . . 19

2.2.1 Dados com Censura Tipo I . . . . . . . . . . . . . . . 19

2.2.2 Dados com Censura Tipo II . . . . . . . . . . . . . 21

2.3 Modelo Misto sob Censura Tipo II . . . . . . . . . . . . . . 23

2.3.1 Teste de Hipótese . . . . . . . . . . . . . . . . 26

2.3.2 Análise do Ajuste do Modelo . . . . . . . . . . . . 28

2.3.3 Multiplas Unidades Amostrais . . . . . . . . . . . . 29

3 Aplicações 33

3.1 Estudo de Caso I . . . . . . . . . . . . . . . . . . . . . . . . 33

3.2 Estudo de Caso II . . . . . . . . . . . . . . . . . . . 37

3.3 Estudo de Caso III . . . . . . . . . . . . . . . . . . . . . 40

3.4 Considerações Finais . . . . . . . . . . . . . . . . 45 


\section{Lista de Figuras}

3.1 Comparação de Ajustes - USS Grampus . . . . . . . . . . 35

3.2 Modelo Ajustado - USS Grampus . . . . . . . . . . . . 36

3.3 QQ Plot dos Resíduos - USS Grampus . . . . . . . . . . 37

3.4 Comparação de Ajustes - USS Halfbeak . . . . . . . . . . . . 39

3.5 Modelo Ajustado - USS Halfbeak . . . . . . . . . . . 40

3.6 QQ Plot dos Resíduos - USS Halfbeak . . . . . . . . . . 41

3.7 Incidência de Falhas por Sistema . . . . . . . . . . . 42

3.8 Modelo Ajustado - Sistemas . . . . . . . . . . . . . . . 43

3.9 QQ Plot dos Resíduos - Sistemas . . . . . . . . . . . . 44 


\section{CAPÍTUlo 1}

\section{Processos Pontuais}

Existem, basicamente, dois tipos de modelos que são aplicados na análise de sistemas reparáveis. Os modelos estocásticos baseados em processos pontuais e os modelos determinísticos baseados em sistemas de equações diferenciais.

Devido à sua importância no estudo da confiabilidade de sistemas reparáveis, iremos abordar, neste trabalho, apenas os modelos baseados em processos pontuais. Não daremos ênfase, entretanto, aos aspectos teóricos relacionados com a sua construção e caracterização. Para esses detalhes o leitor pode recorrer à $[2,11,28]$.

Os modelos baseados em sistemas de equações diferenciais, utilizados principalmente na modelagem do crescimento da confiabilidade de produtos em desenvolvimento, estão fora do escopo deste trabalho. Existe, entretanto, uma vasta literatura que trata deste tópico. Para uma primeira abordagem desses tópicos o leitor pode recorrer à $[2,13]$.

Os processos pontuais são, de uma maneira geral, modelos probabilísticos que descrevem a distribuição aleatória de pontos em um conjunto $S$. Do ponto de vista formal, o modelo é descrito por um espaço de probabilidades $(\Omega, F, P)$ em que o espaço amostral é o conjunto de todas as funções definidas em $S$ com valores inteiros não negativos, isto é, $\Omega=\mathbb{N}^{S}$. Para um particular elemento $\omega \in \Omega, \omega(s), s \in S$, representa o número de pontos que, após a distribuição aleatória em $S$, ocupam a posição $s$. 
Exemplo: Suponha que desejamos construir um modelo probabilístico para descrever a distribuição espacial de acidentes em cruzamentos da malha viária de uma cidade em um determinado dia. Neste caso, é natural tomarmos $S$ como o conjunto de todos os cruzamentos da malha viária desta cidade. Uma particular função do espaço amostral $\Omega=\mathbb{N}^{S}$, representará o número de acidentes em cada um destes cruzamentos em um dia. Em particular, $\{s \in S: \omega(s)>0\}$, é conjunto dos cruzamentos em que ocorreram acidentes nesse dia.

Neste contexto a $\sigma$-álgebra dos eventos é definida como a $\sigma$-álgebra gerada pelas projeções $\{\omega \in \Omega: \omega(s)=k\}, s \in S$ e,$k \in \mathbb{N}$. Observe que, com essa estrutura, para cada $s \in S$, o número de pontos que, após a distribuição aleatória em $S$, ocupam a posição $s$ é a variável aleatória discreta $X_{s}: \Omega \rightarrow \mathbb{N}$, definida por $X_{s}(\omega)=\omega(s)$. Em particular, a distribuição de probabilidade de $X_{s}, s \in S$, é dada por $\omega_{s}(k)=P\left\{X_{s}=k\right\}=P\{\omega \in \Omega: \omega(s)=k\}$. Para cada $\omega \in \Omega$ fixado, a função $s \in S \rightarrow X_{s}(\omega)=\omega(s)$, é chamada de uma trajetória do processo pontual $(\Omega, F, P)$.

Exemplo: No exemplo anterior, a variável aleatória $X_{s}, s \in S$ fixado, descreve o número de acidentes no cruzamento $s$ da malha viária da cidade. $\triangle$

Na modelagem probabilística de sistemas ou equipamentos reparáveis, os processos pontuais são utilizados para descrever o comportamento dinâmico dos instantes em que suas falhas ocorrem ao longo do tempo. Nesse contexto, o espaço amostral desses processos é definido como $\Omega=\mathbb{N}^{(0, \infty)}$ e para cada $\omega \in \Omega$ fixado $\{t \in[0, \infty): \omega(t)>0\}$ representa o conjunto dos instantes em que as falhas ocorrem. Entretanto, as medidas de probabilidade utilizadas nesse contexto estão concentradas no conjunto $\Omega_{0}=\{0,1\}^{[0, \infty)}$, isto é, $P\left\{\Omega_{0}\right\}=1$. Processos pontuais com essa propriedade são denominados simples ou sem pontos múltiplos. Neste trabalho nos restringiremos aos processos pontuais deste tipo e descreveremos de que maneira famílias especiais destes processos são utilizadas nos estudos da confiabilidade de sistemas ou equipamentos reparáveis. Nesses estudos, entretanto, costuma-se utilizar uma caracterização alternativa, tomando-se como espaço amostral o 
conjunto $\Omega$ das funções $f:[0, \infty) \rightarrow \mathbb{N}$, tais que:

1. $f$ é monótona crescente, não limitada e contínua à direita;

2. $f(0)=0$;

3. $0 \leq f(t)-f\left(t_{-}\right) \leq 1$ para $t \in[0, \infty)$.

Para um processo pontual $(\Omega, F, P)$ com o espaço amostral descrito acima, denota-se por $N_{t}, t \geq 0$, a variável aleatória discreta definida em $\Omega$ por $N_{t}(\omega)=\omega(t), \quad t \in[0, \infty)$. Essa variável representa o número de pontos distribuídos ao acaso em $[0, \infty)$, contídos no intervalo $[0, t]$. Além disso, para cada $\omega$ fixado, a função $t \rightarrow N_{t}(\omega)$ é monótona crescente, não limitada, contínua à direita, crescendo apenas por saltos de tamanho igual a $1 \mathrm{e}$ $N_{0}(\omega)=0$. De uma maneira geral, o número de pontos contidos em um subconjunto de Borel $A \subset[0, \infty)$ é dado pela variável aleatória definida por $N_{A}=\int_{A} d N_{t}$.

No contexto dos sistemas ou equipamentos reparáveis a variável aleatória $N_{t}$ denota o número de falhas ocorridas até o instante $t$. Em particular, para $0 \leq t_{1}<t_{2}, N_{\left(t_{1}, t_{2}\right]}=\int_{\left(t_{1}, t_{2}\right]} d N_{t}=N_{t_{2}}-N_{t_{1}}$, representa o número de falhas ocorridas no intervalo $\left(t_{1}, t_{2}\right]$.

A família de variáveis aleatórias inteiras, $\left\{N_{t}: t \geq 0\right\}$ introduzida acima, é denominada processo de contagem associado ao processo pontual $(\Omega, F, P) \mathrm{e}$ não é dificil ver que é possível definir um processo pontual simples a partir de um processo com as mesmas características estruturais de $\left\{N_{t}: t \geq 0\right\}$. Para os objetivos do presente trabalho, adotaremos este caminho. Formalmente, têm-se:

Definição 1.1: Um processo pontual simples é uma família $\left\{N_{t}: t \geq 0\right\}$ de variáveis aleatórias inteiras definidas em um espaço de probabilidades $(\Omega, F, P)$, tal que para cada $\omega \in \Omega$, a função $f_{\omega}:[0, \infty) \rightarrow \mathbb{N}$ definida por $f_{\omega}(t)=N_{t}(\omega)$ satisfaz as propriedades de 1 a 3 acima.

Se $\left\{N_{t}: t \geq 0\right\}$ é um processo pontual simples, não é difícil ver que as variáveis aleatórias não negativas definidas por $T_{n}=\inf \left\{t \geq 0: N_{t} \geq n\right\}$, $n \geq 0$, tem as seguintes propriedades: 
1. $T_{0}=0$

2. $\left\{T_{n} ; n \geq 0\right\}$ é monótona crescente, isto é, $T_{0} \leq T_{1} \leq \ldots \leq T_{n}$

3. $\lim _{n \rightarrow \infty} T_{n}=+\infty$

Essas variáveis indicam os instantes em que ocorrem saltos na trajetória de $\left\{N_{t}: t \geq 0\right\}$. A seqüência $\left\{T_{n}: n \geq 0\right\}$ é comumente denominada processo de chegadas associado ao processo pontual $\left\{N_{t}: t \geq 0\right\}$. No contexto dos sistemas ou equipamentos reparáveis as variáveis aleatórias $T_{n}, n \geq 1$, representam os instantes em que as sucessivas falhas de um sistema ou equipamento ocorrem.

Se $\left\{T_{n}: n \geq 0\right\}$ é o processo de chegadas associado a um processo pontual $\left\{N_{t}: t \geq 0\right\}$ e a distribuição condicional de $U_{n}=T_{n+1}-T_{n}$ dado $T_{0}, T_{1}, \ldots, T_{n}$ tem taxa de falha $h_{n}\left(t: T_{0}, T_{1}, \ldots, T_{n}\right)$, então

$$
\lim _{s \rightarrow 0} \frac{1}{s} P\left\{N_{(t, t+s]} \geq 1 \mid N_{u}: u<t\right\}=h_{N_{t-}}\left(t: T_{0}, T_{1}, \ldots, T_{N_{t-}}\right)
$$

O lado direito de (1.1) define o que na teoria dos processos pontuais costuma-se chamar de função intensidade completa de $\left\{N_{t}: t \geq 0\right\}$. Especificamente:

Definição 1.2: Para um processo pontual simples $\left\{N_{t}: t \geq 0\right\}$, a função $\lambda\left(t \mid N_{u}: u<t\right), t \geq 0$, definida por

$$
\lambda\left(t \mid N_{u}: u<t\right)=\lim _{s \rightarrow 0} \frac{1}{s} P\left\{N_{(t, t+s]} \geq 1 \mid N_{u}: u<t\right\}
$$

é denominada função intensidade completa do processo.

Conforme [12], proposição 7.2.IV, para os processos pontuais simples a função intensidade completa determina, de maneira única, a sua distribuição de probabilidades. A construção de processos pontuais a partir de suas funções intensidades completas, entretanto, não é comum na literatura. Para maiores detalhes o leitor pode consultar as referências $[3,12]$.

No contexto dos sistemas ou equipamentos reparáveis, duas outras funções são também utilizadas para caracterizar o fluxo de falhas ao longo do tempo. São elas: 
1. A função ROCOF ou taxa de ocorrência de falhas e

2. A função intensidade de falhas,

que são definidas da seguinte maneira:

Definição 1.3: A função média acumulada, $\Lambda(t), t \geq 0$, de um processo pontual $\left\{N_{t}: t \geq 0\right\}$ é definida por

$$
\Lambda(t)=E\left(N_{t}\right)
$$

Se $\Lambda(t)$ for diferenciável, sua derivada, $\mu(t), t \geq 0$, é denominada função ROCOF (taxa de ocorrência de falhas) de $\left\{N_{t}: t \geq 0\right\}$, isto é,

$$
\mu(t)=\frac{\partial \Lambda(t)}{\partial t}
$$

No contexto dos sistemas ou equipamentos reparáveis a função ROCOF nos dá, portanto, a taxa instantânea de crescimento do número esperado de falhas.

Definição 1.4: A função intensidade de falhas, $\lambda(t), t \geq 0$, de um processo pontual $\left\{N_{t}: t \geq 0\right\}$ é definida por

$$
\lambda(t)=\lim _{s \rightarrow 0} \frac{1}{s} P\left(N_{(t, t+s]} \geq 1\right)
$$

A função intensidade de falhas de um processo pontual mede a taxa instantânea de crescimento da probabilidade de falhas. A função ROCOF e a função intensidade de falhas de um processo pontual $\left\{N_{t}: t \geq 0\right\}$ são geralmente distintas. Para processos simples, entretanto, as duas funções são iguais, como pode ser verificado na Proposição 1.

Proposição 1: Se $\left\{N_{t}: t \geq 0\right\}$ é um processo pontual simples, então $\mu(t)=\lambda(t)$, para todo $t \geq 0$. 
Prova: Como visto na Definição $1.3, \Lambda(t)=E\left(N_{t}\right)$, logo podemos escrever que

$$
\begin{aligned}
& \Lambda(t+s)-\Lambda(t)=E\left(N_{(t, t+s]}\right) \Rightarrow \frac{\Lambda(t+s)-\Lambda(t)}{s}=\frac{E\left(N_{(t, t+s]}\right)}{s} \\
& \Rightarrow \lim _{s \rightarrow 0} \frac{\Lambda(t+s)-\Lambda(t)}{s}=\lim _{s \rightarrow 0} \frac{E\left(N_{(t, t+s]}\right)}{s}
\end{aligned}
$$

o lado esquerdo da equação acima fica dado por $\lim _{s \rightarrow 0} \frac{\Lambda(t+s)-\Lambda(t)}{s}=\mu(t)$, já para o lado direito temos que

$$
\lim _{s \rightarrow 0} \frac{E\left(N_{(t, t+s]}\right)}{s}=\lim _{s \rightarrow 0} \frac{\sum_{k=1}^{\infty} P\left(N_{(t, t+k]} \geq k\right)}{s}
$$

reescrevendo a última parte da equação tem-se

$\lim _{s \rightarrow 0} \frac{\sum_{k=1}^{\infty} P\left(N_{(t, t+k]} \geq k\right)}{s}=\lim _{s \rightarrow 0} \frac{P\left(N_{(t, t+k]} \geq 1\right)}{s}+\lim _{s \rightarrow 0} \frac{\sum_{k=2}^{\infty} P\left(N_{(t, t+k]} \geq k\right)}{s}$

mas

$$
\lim _{s \rightarrow 0} \frac{P\left(N_{(t, t+k]} \geq 1\right)}{s}=\lambda(t) e \lim _{s \rightarrow 0} \frac{\sum_{k=2}^{\infty} P\left(N_{(t, t+k]} \geq k\right)}{s}=o(s)
$$

$\operatorname{logo} \mu(t)=\lambda(t)$ o que completa a nossa prova. $\triangle$

\subsection{Processos Pontuais Simples}

Duas famílias de processos pontuais simples têm importância histórica na modelagem probabilística de sistemas ou equipamentos reparáveis. São elas:

1. A família dos processos pontuais de Poisson e

2. A família dos processos pontuais de Renovação.

Essas duas famílias de modelos facilitam, respectivamente, o tratamento de duas situações típicas com respeito ao efeito da ação de manutenção sobre o sistema ou equipamento de interesse: Uma em que ela não retorna o sistema à condição de tão bom quanto novo, e em que se costuma modelar os instantes de falhas ao longo do tempo através de um processo pontual de Poisson, e outra em que a ação restauradora é completa e o modelo natural para esses instantes é um processo pontual de renovação. Formalmente, tem-se: 
Definição 1.5: Diz-se que um processo pontual simples $\left\{N_{t}: t \geq 0\right\}$ é de Poisson se sua função intensidade completa, $\lambda\left(t \mid N_{u}: u<t\right), t \geq 0$, é da forma

$$
\lambda\left(t \mid N_{u}: u<t\right)=\rho(t) .
$$

Se $\rho(t)=\rho$, para todo $t \geq 0$, diz-se ainda que o processo pontual de Poisson é homogêneo.

Definição 1.6: Diz-se que um processo pontual simples $\left\{N_{t}: t \geq 0\right\}$ é de renovação se sua função intensidade completa, $\lambda\left(t \mid N_{u}: u<t\right), t \geq 0$, é da forma

$$
\lambda\left(t \mid N_{u}: u<t\right)=\rho\left(t-T_{N_{t-}}\right),
$$

em que $T_{N_{t-}}$ é o instante do último salto na trajetória do processo, antes do instante t.

Essas famílias de processos têm propriedades matemáticas interessantes e são estudadas com detalhes em [9]. Não custa lembrar, entretanto, que:

\section{Proposição 2:}

1. Um processo pontual simples $\left\{N_{t}: t \geq 0\right\}$ é de Poisson se e somente se tiver incrementos independentes, isto é, se para quaisquer $s, t \geq 0, N_{(t, t+s]}$ for independente de $\left\{N_{u}: u \leq t\right\}$;

2. Um processo pontual de Poisson $\left\{N_{t}: t \geq 0\right\}$ é homogêneo se e somente se tiver incrementos estacionários, isto é, se para quaisquer $s, t \geq 0$, a distribuição de $N_{(t, t+s]}$ for independente de $t$.

3. Para um processo pontual de Poisson $\left\{N_{t}: t \geq 0\right\}$ com função média acumulada contínua $\Lambda,\left\{M_{t}: t \geq 0\right\}$ definido por $M_{t}=N_{\tau(t)}$, em que

$$
\tau(t)=\inf \{s: \Lambda(s)>t\}, t \geq 0,
$$

é um processo pontual de Poisson homogêneo com função ROCOF constante e igual a 1. Além disso, para quaisquer $s, t \geq 0$,

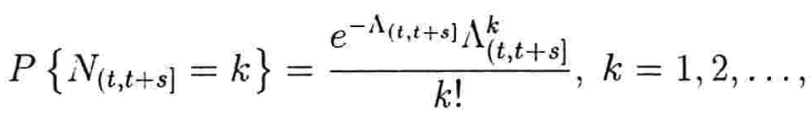

em que $\Lambda_{(t, t+s]}=\Lambda(t+s)-\Lambda(t)$. 
Se $\left\{T_{n}: n \geq 1\right\}$ é o processo de chegadas associado a $\left\{N_{t}: t \geq 0\right\}$, dado $\left\{N_{t}=k\right\}, T_{1}<T_{2}<\ldots<T_{N_{t}}$ tem a distribuição conjunta das estatísticas de ordem de uma amostra casual simples de tamanho $k-1$ da distribuição

$$
G(y)= \begin{cases}0 & \text { se } y \leq 0 \\ \frac{\Lambda(y)}{\Lambda(t)} & \text { se } 0<y \leq t \\ 1 & \text { se } y>t\end{cases}
$$

Se

$$
\Lambda(t)=\int_{0}^{t} \lambda(x) d x
$$

a densidade conjunta de $T_{1}<T_{2}<\ldots<T_{n}$, para $n \geq 1$ fixado, é dada por

$$
f\left(t_{1}, t_{2}, \ldots, t_{n}\right)=\left(\prod_{i=1}^{n} \lambda\left(t_{i}\right)\right) e^{-\int_{0}^{t_{n}} \lambda(x) d x}, 0 \leq t_{1}<t_{2}<\ldots<t_{n} .
$$

4. Para um processo pontual de renovação $\left\{N_{t}: t \geq 0\right\}$, os tempos $W_{n}=$ $T_{n}-T_{n-1}, n \geq 1$, entre os instantes $T_{n}=$ inf $\left\{t \geq 0: N_{t} \geq n\right\}, n \geq 1$, em que ocorrem saltos na sua trajetória, são variáveis aleatórias independentes e identicamente distribuídas com taxa de falha $\rho(t)$.

Como já discutido no início deste capítulo, os processos pontuais simples de Poisson e de Renovação não são geralmente definidos na literatura através da forma de sua função intensidade completa. Optamos por utilizar esta abordagem apenas para facilitar, no contexto da análise da confiabilidade de sistemas ou equipamentos reparáveis, o tratamento unificado dessas duas famílias de modelos. Para tanto, introduziremos uma terceira família de modelos - os modelos mistos - que incluem as duas famílias anteriormente descritas como casos particulares.

Definição 1.7: Diz-se que um processo pontual simples $\left\{N_{t}: t \geq 0\right\}$ é misto se sua função intensidade completa, $\lambda\left(t \mid N_{u}: u<t\right), t \geq 0$, é da forma

$$
\lambda\left(t \mid N_{u}: u<t\right)=\exp \left\{\Theta^{T} \mathbf{Z}\left(t \mid N_{u}: u<t\right)\right\},
$$

em que $\Theta^{T}=\left(\theta_{1}, \ldots, \theta_{k}\right)$ é um vetor de parâmetros e

$$
\mathbf{Z}^{T}\left(t \mid N_{u}: u<t\right)=\left(Z_{1}\left(t \mid N_{u}: u<t\right), \ldots, Z_{k}\left(t \mid N_{u}: u<t\right)\right)
$$


um vetor de funções intensidades completas.

Vários modelos especiais são casos particulares da função intensidade completa descrita em (1.10):

- Um processo de Poisson com função intensidade log-linear, isto é, $\rho(t)=$ $\exp \{\alpha+\beta t\}, t \geq 0$, é obtido tomando-se

$$
\Theta=\left(\begin{array}{c}
\alpha \\
\beta
\end{array}\right), \mathbf{Z}\left(t \mid N_{u}: u<t\right)=\left(\begin{array}{c}
1 \\
t
\end{array}\right)
$$

- Um processos de Poisson com função intensidade power-law, isto é, $\rho(t)=\alpha t^{\beta}$, é obtido tomando-se

$$
\Theta=\left(\begin{array}{c}
\ln \alpha \\
\beta
\end{array}\right), \mathbf{Z}\left(t \mid N_{u}: u<t\right)=\left(\begin{array}{c}
1 \\
\ln t
\end{array}\right)
$$

- Um processos de renovação com distribuição Weibull com taxa de falha $\rho(t)=\alpha t^{\beta}$ entre renovações é obtido tomando-se

$$
\Theta=\left(\begin{array}{c}
\ln \alpha \\
\beta
\end{array}\right), \mathbf{Z}\left(t \mid N_{u}: u<t\right)=\left(\begin{array}{c}
1 \\
\ln \left(t-T_{N_{t-}}\right)
\end{array}\right)
$$

No contexto da análise da confiabilidade de um sistema ou equipamento reparável, nosso interesse consiste em utilizar como base um modelo flexível que misture aspectos poissonianos e de renovação ao invés de considerá-los de forma isolada em modelos específicos. Assim, no próximo capítulo, enfatizaremos a análise da confiabilidade de um sistema ou equipamento reparável, a partir de um conjunto de dados de falha, tomando como base um modelo misto em que

$$
\mathbf{Z}\left(t \mid N_{u}: u<t\right)=\left(\begin{array}{c}
1 \\
\rho_{1}(t) \\
\rho_{2}\left(t-T_{N_{t-}}\right)
\end{array}\right)
$$

para escolhas particulares de $\rho_{1}$ e $\rho_{2}$. 


\subsection{Outros Processos Pontuais}

É importante ressaltar que existem outros processos pontuais que são amplamente utilizados em análises de confiabilidade de sistemas reparáveis, mas que fogem ao escopo deste trabalho.

Dizer que os tempos entre sucessivas falhas, $X_{1}, X_{2}, \ldots, X_{n}$, são variáveis aleatórias independentes com distribuição exponencial, não implica necessariamente em termos um processo de Poisson, pois isto só seria verdade se estes tempos também fossem identicamente distribuídos. O relaxamento do fato de serem identicamente distribuídos permite que o tempo entre falhas possa ter distribuições exponenciais com médias distintas. Neste caso [33] propuseram um modelo, o qual chamaram de Piecewise Exponential $(P E X P)$. Neste modelo eles assumiram que $X_{1}, X_{2}, \ldots, X_{n}$ são variáveis aleatórias independentes com

$$
E\left(X_{i}\right)=\frac{\delta i^{\delta-1}}{\mu}, \text { para } i=1,2, \ldots
$$

onde $\delta>0$ e $\mu>0$. Vale a pena notar que :

$$
\delta \begin{cases}<1 & \text { Sistema está deteriorando, } \\ =1 & \text { Sistema estável - (Processo de Poisso Homogêneo) } \\ >1 & \text { Sistema está amadurecendo (melhorando) }\end{cases}
$$

Enquanto que os modelos de Poisson Não Homogêneos pressupõem que a taxa de falhas varia continuamente no decorrer do tempo, os modelos baseados em PEXP assumem que a taxa de falhas do sistema mantém-se constante entre duas falhas sucessivas ocorrendo saltos apenas nos instantes de falha. Assim, nos estudos de crescimentos de confiabilidade, onde as melhorias são introduzidas após a ocorrência de uma falha, os modelos PEXP são mais apropriados que os modelos de Poisson Não Homogêneos.

Outro modelo importante, proposto em [10], são os chamados processos de renovação modulados $(P R M)$. Esses processos tem função intensidade completa dada por

$$
\lambda\left(t \mid N_{u}: u<t\right)=h\left(t-T_{N_{t-}}\right) e^{\beta_{1} z_{1}(t)+\ldots+\beta_{p} z_{p}(t)} .
$$


Note que se $\beta_{1}=\beta_{2}=\ldots=\beta_{p}=0$, então $\lambda\left(t \mid N_{u}: u<t\right)=h\left(t-T_{N_{t-}}\right)$, e neste caso o processo reduz-se a um processo de renovação. Se $h\left(t-T_{N_{t-}}\right)$ for constante o processo reduz-se a um processos de Poisson Não Homogêneo. Portanto um processo de renovação Modulado incorpora tanto os processos de Poisson quanto os de Renovação. Dependendo de como a função intensidade completa é escrita em função dos $z_{i}(t)$, pode-se modelar funções voltadas principalmente para

- Tendências na intensidade de ocorrência das falhas,

- Fenônemos periódicos,

- Fatores de Carga ou Stress.

Uma análise mais detalhada desses processos pode ser encontrada em [10] e [28]. Um caso particular interessante é dos processos gama não homogêneos, introduzidos em [6].

Em alguns casos existe a tendência das falhas ocorrerem umas próximas das outras. Isto pode indicar que o sistema não foi reparado corretamente, gerando uma série de novas falhas. Este fato é mais observado em sistemas eletrônicos e mecânicos. Como exemplo, considere uma placa de circuito de segurança que apresentou uma falha no instante $t$. Um técnico em eletrônica analisou o circuito e diagnosticou que o sistema falhou devido a um regulador de tensão que deveria estar mantendo constante uma corrente de 3 Volts para alimentar o sistema. Entretanto o regulador estava osciliando, com a corrente chegando a picos de 14 Volts. Por isso, o regulador de tensão induziu a falha em um transístor e um diodo. Se o primeiro componente (regulador de tensão) não falhasse, então não ocorreria as falhas do transístor e do diodo. Modelos para este tipo de situação foram introduzidos por Bartlett, para modelar o fluxo de trânsito em uma rodovia, onde a lentidão de um veículo poderia gerar, imediatamente, a lentidão de outros. Este modelo logo foi extrapolado para o caso onde uma simples falha em um sistema fosse seguido por outras falhas. Detalhes sobre esses processos podem ser encontrados em [5]. 
Por fim, um outro modelo que não poderíamos deixar de lado, são os modelos com reparos imperfeitos, sugeridos por Borges em [7]. Este modelo pressupõe que após uma falha no instante $t$ o sistema pode ser reparado com um reparo perfeito, com probabilidade $p(t)$, ou com um reparo mínimo, com probabilidade $q(t)=1-p(t)$. Casos especiais são obtidos quando $p=0$, onde temos um processos de Posisson Não Homogêneo, ou quando $p=1$ onde temos um processo de Renovação. 


\section{CAPÍtulo 2}

\section{Inferência Estatística dos Modelos}

No capítulo anterior foram introduzidas a noção de processos pontuais simples e as definições das famílias modelos tipicamente utilizadas em estudos da confiabilidade de sistemas ou equipamentos reparáveis bem como as de maior interesse nesse trabalho. Neste capítulo, trataremos especificamente das questões inferenciais para essas famílias. Embora nosso interesse se concentre nos modelos mistos, incluimos também nesse capítulo, devido à sua importância histórica, o tratamento inferencial para os modelos de Poisson com funções intensidade power-law e log-linear. O tratamento do modelo misto baseia-se no trabalho de Lawless e Thiagorajah publicados em [20]. Além disso, trataremos apenas o caso de uma única unidade de observação. Uma pequena discussão sobre o caso de múltiplas unidades de observação é feita no final deste Capítulo.

\subsection{Modelo Power-Law}

Sob o modelo power-law, os instantes de falha experimentados pelo sistema se comportam como o processo de chegadas, $\left\{T_{n}: n \geq 1\right\}$, de um processo pontual de Poisson, $\left\{N_{t}: t \geq 0\right\}$, com função intensidade completa dada por

$$
\lambda\left(t \mid N_{u}: u<t\right)=\frac{\beta}{\theta}\left(\frac{t}{\theta}\right)^{\beta-1}, \text { para } \theta, \quad \beta \in(0, \infty) .
$$

Nesse modelo, o parâmetro $\beta$, conhecido como parâmetro de forma, indica o tipo de relação entre o tempo e a intensidade de falhas. Três casos 
configuram aspectos qualitativos distintos da intensidade de falhas ao longo do tempo:

- Wear-in: $\beta<1$

- Wear-less: $\beta=1$

- Wear-out: $\beta>1$

Quando $\beta=1$, a função intensidade de falhas é constante e igual a $1 / \theta$ e as falhas ocorrem ao longo do tempo segundo um processo de Poisson homogêneo. Quando $\beta<1$, espera-se que com o decorrer do tempo as falhas experimentadas pelo sistema se tornem cada vez mais esparsas (menos freqüêntes). Nesse caso dizemos que o sistema está amaciando. Quando $\beta>1$, espera-se que com o decorrer do tempo as falhas experimentadas pelo sistema se acumulem, tornando-se cada vez menos esparsas.

O parâmetro $\theta$, por sua vez, é conhecido como parâmetro de escala. Para valores fixados do tempo e do parâmetro $\beta$, quanto maior o valor do parâmetro $\theta$, menor será o número esperado de falhas do sistema.

Em determinadas situações, pode ser conveniente utilizar uma parametrização alternativa para o modelo Power-Law. Uma reparametrização comumente utilizada é:

$$
\lambda\left(t \mid N_{u}: u<t\right)=\gamma \beta t^{\beta-1}
$$

Para esta reparametrização a interpretação do parâmetro $\beta$ permanece a mesma e $\gamma=(1 / \theta)^{\beta}$.

\subsubsection{Dados com Censura Tipo I}

Sob censura tipo I, o sistema é observado até um instante final pré-fixado t. Neste caso, tanto o número de falhas, $N_{t}$, quanto os instantes $T_{1}<T_{2}<$ $\ldots<T_{N_{t}}<t$ em que elas ocorrem, são variáveis aleatórias. Além disso, pela proposição 2 item $3, N_{t}$ tem distribuição de Poisson com média $\Lambda(t)=(t / \theta)^{\beta}$ e, dado $N_{t}=k, k \geq 1, T_{1}<T_{2}<\ldots<T_{N_{t}}<t$ têm a distribuição conjunta 
das estatísticas de ordem de uma amostra casual simples de tamanho $k$ da distribuição

$$
G(y)= \begin{cases}0 & \text { se } y \leq 0 \\ \left(\frac{y}{t}\right)^{\beta} & \text { se } 0<y \leq t \\ 1 & \text { se } y>t\end{cases}
$$

Assim, a densidade conjunta de $N_{t}, T_{1}, T_{2}, \ldots, T_{N_{t}}$ é dada por:

$f\left(t_{1}, t_{2}, \ldots, t_{n}, n\right)= \begin{cases}f_{N}(n) f\left(t_{1}, t_{2}, \ldots, t_{n} \mid n\right) & \text { se } n \geq 1 \\ & \text { e } 0 \leq t_{1}<t_{2}<\ldots<t_{n} \\ f_{N}(0) & \text { se } n=0\end{cases}$ em que

$$
f_{N}(n)=\frac{\left[(t / \theta)^{\beta}\right]^{n} e^{-(t / \theta)^{\beta}}}{n !}, \quad n=0,1, \ldots
$$

$\mathrm{e}$

$$
f\left(t_{1}, t_{2}, \ldots, t_{n} \mid n\right)=n ! \prod_{i=1}^{n} \frac{\beta}{t}\left(\frac{t_{i}}{t}\right)^{\beta-1}, n \geq 1 \text { e } 0 \leq t_{1}<t_{2}<\ldots<t_{n} .
$$

Portanto,

$$
f\left(t_{1}, t_{2}, \ldots, t_{n}, n\right)= \begin{cases}\frac{\beta^{n}}{\theta^{n \beta}} e^{-(t / \theta)^{\beta}} \prod_{i=1}^{n} t_{i}^{\beta-1} & \text { se } n \geq 1 \\ & \text { e } 0 \leq t_{1}<t_{2}<\ldots<t_{n} \\ e^{-(t / \theta)^{\beta}} & \text { se } n=0\end{cases}
$$

Se $n \geq 1$, que é, obviamente, o caso de maior interesse, o logaritimo da função de verossimilhança é dado por

$$
\begin{aligned}
l(\beta, \theta ; n, \mathrm{~T}) & =n \ln \frac{\beta}{\theta^{\beta}}+(\beta-1) \sum_{i=1}^{n} \ln \frac{t_{i}}{\theta}-\left(\frac{t}{\theta}\right)^{\beta} \\
& =n \ln \beta-n \beta \ln \theta+(\beta-1) \sum_{i=1}^{n} \ln t_{i}-\left(\frac{t}{\theta}\right)^{\beta},
\end{aligned}
$$

e os estimadores de máxima verossimilhança para os parâmetros $\beta$ e $\theta$, são obtidos resolvendo o seguinte sistema não-linear de equações: 


$$
\begin{aligned}
& \frac{l(\beta, \theta ; \mathbf{X})}{\partial \beta}=\frac{n}{\beta}-n \ln \theta+\sum_{i=1}^{n} \ln t_{i}-\left(\frac{t}{\theta}\right)^{\beta} \ln \left(\frac{t}{\theta}\right)=0 \\
& \frac{l(\beta, \theta ; \mathbf{X})}{\partial \theta}=-\frac{n \beta}{\theta}+\left(\frac{t}{\theta}\right)^{\beta} \frac{\beta}{\theta}=0 .
\end{aligned}
$$

Da equação (2.3) obtêm-se:

$$
\begin{aligned}
-n+\left(\frac{t}{\theta}\right)^{\beta}=0 & \Rightarrow\left(\frac{t}{\theta}\right)^{\beta}=n \\
& \Rightarrow \ln \left(\frac{t}{\theta}\right)=\frac{1}{\beta} \ln n \\
& \Rightarrow \ln t-\ln \theta=\ln n^{1 / \beta} \\
& \Rightarrow \ln t-\ln n^{1 / \beta}=\ln \theta \\
& \Rightarrow \ln \frac{t}{n^{1 / \beta}}=\ln \theta \\
& \Rightarrow \theta=\frac{t}{n^{1 / \beta}} .
\end{aligned}
$$

Substituindo o resultado acima na equação (2.2) e resolvendo em relação a $\beta$, têm-se:

$$
\begin{aligned}
0 & =\frac{n}{\beta}-n \ln \frac{t}{n^{1 / \beta}}+\sum_{i=1}^{n} \ln t_{i}-\left(\frac{t}{\frac{t}{n^{1 / \beta}}}\right)^{\beta} \ln \left(\frac{t}{\frac{t}{n^{1 / \beta}}}\right) \\
& =\frac{n}{\beta}-n \ln \frac{t}{n^{1 / \beta}}+\sum_{i=1}^{n} \ln t_{i}-n \ln n^{1 / \beta} \\
& =\frac{n}{\beta}-n \ln t+n \ln n^{1 / \beta}+\sum_{i=1}^{n} \ln t_{i}-n \ln n^{1 / \beta} \\
& =\frac{n}{\beta}-n \ln t+\sum_{i=1}^{n} \ln t_{i} \\
& =\frac{n}{\beta}-\sum_{i=1}^{n} \ln t+\sum_{i=1}^{n} \ln t_{i} \\
& =\frac{n}{\beta}-\sum_{i=1}^{n} \ln \frac{t}{t_{i}} \Rightarrow \beta=\frac{n}{\sum_{i=1}^{n} \ln \frac{t}{t_{i}}}
\end{aligned}
$$

Portanto, os estimadores de máxima verossimilhança para $\theta$ e $\beta$ são dados 
por:

$$
\begin{aligned}
\hat{\theta}\left(T_{1}, T_{2}, \ldots, T_{N_{t}}, N_{t}\right) & =\frac{t}{n^{1 / \hat{\beta}\left(T_{1}, T_{2}, \ldots, T_{N_{t}}, N_{t}\right)}} \\
\hat{\beta}\left(T_{1}, T_{2}, \ldots, T_{N_{t}}, N_{t}\right) & =\frac{n}{\sum_{i=1}^{n} \ln \frac{t}{t_{i}}} .
\end{aligned}
$$

\subsubsection{Dados com Censura Tipo II}

Sob censura tipo II, o sistema é observado até a ocorrência da n-ésima falha, isto é, até o instante $T_{n}$. Neste caso, segue da proposição 2 item 3 que

$$
T_{1}<T_{2}<\ldots<T_{n}
$$

tem densidade conjunta dada por

$$
\begin{aligned}
f\left(t_{1}, t_{2}, \ldots, t_{n}\right) & =\prod_{i=1}^{n} \lambda\left(t_{i}\right) e^{-\int_{0}^{t_{n}} \lambda(x) d x} \\
& =\prod_{i=1}^{n} \frac{\beta}{\theta}\left(\frac{t_{i}}{\theta}\right)^{\beta-1} e^{-\left(\frac{t_{n}}{\theta}\right)^{\beta}}, \quad 0 \leq t_{1}<t_{2}<\ldots<t_{n} .
\end{aligned}
$$

O Logaritimo da função de verossimilhança, nesse caso, é dado por:

$$
\begin{aligned}
l(\beta, \theta ; \mathrm{T}) & =\sum_{i=1}^{n} \ln \frac{\beta}{\theta}+\sum_{i=1}^{n}(\beta-1) \ln \frac{t_{i}}{\theta}-\left(\frac{t_{n}}{\theta}\right)^{\beta} \\
& =n \ln \frac{\beta}{\theta}+(\beta-1) \sum_{i=1}^{n} \ln \frac{t_{i}}{\theta}-\left(\frac{t_{n}}{\theta}\right)^{\beta} \\
& =n \ln \beta-n \ln \theta+(\beta-1) \sum_{i=1}^{n}\left(\ln t_{i}-\ln \theta\right)-\left(\frac{t_{n}}{\theta}\right)^{\beta} \\
& =n \ln \beta-n \beta \ln \theta+(\beta-1) \sum_{i=1}^{n} \ln t_{i}-\left(\frac{t_{n}}{\theta}\right)^{\beta},
\end{aligned}
$$

e os estimadores de máxima verossimilhança para os parâmetros $\beta$ e $\theta$, são obtidos resolvendo-se o seguinte sistema não-linear de equações

$$
\begin{aligned}
& \frac{l(\beta, \theta ; \mathbf{X})}{\partial \beta}=\frac{n}{\beta}-n \ln \theta+\sum_{i=1}^{n} \ln t_{i}-\left(\frac{t_{n}}{\theta}\right)^{\beta} \ln \left(\frac{t_{n}}{\theta}\right)=0 \\
& \frac{l(\beta, \theta ; \mathbf{X})}{\partial \theta}=-\frac{n \beta}{\theta}+\left(\frac{t_{n}}{\theta}\right)^{\beta} \frac{\beta}{\theta}=0 .
\end{aligned}
$$


Da equação (2.7) têm-se

$$
\begin{aligned}
-n+\left(\frac{t_{n}}{\theta}\right)^{\beta}=0 & \Rightarrow\left(\frac{t_{n}}{\theta}\right)^{\beta}=n \\
& \Rightarrow \ln \left(\frac{t_{n}}{\theta}\right)=\frac{1}{\beta} \ln n \\
& \Rightarrow \ln t_{n}-\ln n^{1 / \beta}=\ln \theta \\
& \Rightarrow \ln \frac{t_{n}}{n^{1 / \beta}}=\ln \theta \\
& \Rightarrow \theta=\frac{t_{n}}{n^{1 / \beta}} .
\end{aligned}
$$

Substituindo o resultado acima na equação (2.6) e resolvendo em relação a $\beta$, tem-se

$$
\begin{aligned}
0 & =\frac{n}{\beta}-n \ln \frac{t_{n}}{n^{1 / \beta}}+\sum_{i=1}^{n} \ln t_{i}-\left(\frac{t_{n}}{\frac{t_{n}}{n^{1 / \beta}}}\right)^{\beta} \ln \left(\frac{t_{n}}{\frac{t_{n}}{n^{1 / \beta}}}\right) \\
& =\frac{n}{\beta}-n \ln \frac{t_{n}}{n^{1 / \beta}}+\sum_{i=1}^{n} \ln t_{i}-n \ln n^{1 / \beta} \\
& =\frac{n}{\beta}-n \ln t_{n}+n \ln n^{1 / \beta}+\sum_{i=1}^{n} \ln t_{i}-n \ln n^{1 / \beta} \\
& =\frac{n}{\beta}-n \ln t_{n}+\sum_{i=1}^{n} \ln t_{i} \\
& =\frac{n}{\beta}-\sum_{i=1}^{n} \ln t_{n}+\sum_{i=1}^{n} \ln t_{i} \\
& =\frac{n}{\beta}-\sum_{i=1}^{n} \ln \frac{t_{n}}{t_{i}} \Rightarrow \beta=\frac{n}{\sum_{i=1}^{n} \ln \frac{t_{n}}{t_{i}}} .
\end{aligned}
$$

Portanto, os estimadores de máxima verossimilhança para $\theta$ e $\beta$ são dados por:

$$
\begin{aligned}
\widehat{\theta}\left(T_{1}, T_{2}, \ldots, T_{n}\right) & =\frac{t_{n}}{n^{1 / \widehat{\beta}\left(T_{1}, T_{2}, \ldots, T_{n}\right)}} \\
\widehat{\beta}\left(T_{1}, T_{2}, \ldots, T_{n}\right) & =\frac{n}{\sum_{i=1}^{n} \ln \frac{t_{n}}{t_{i}}}
\end{aligned}
$$




\subsection{Modelo Log-Linear}

Sob o modelo log-linear, os instantes de falha experimentados pelo sistema se comportam como o processo de chegadas, $\left\{T_{n}: n \geq 1\right\}$, de um processo pontual de Poisson, $\left\{N_{t}: t \geq 0\right\}$, com função intensidade completa dada por

$$
\lambda\left(t \mid N_{u}: u<t\right)=e^{(\alpha+\beta t)}, \text { para } \alpha, \beta \in(-\infty, \infty) .
$$

\subsubsection{Dados com Censura Tipo I}

Sob censura tipo I, o sistema é observado até um instante final pré-fixado $t$. Neste caso, tanto o número de falhas, $N_{t}$, quanto os instantes $T_{1}<T_{2}<$ $\ldots<T_{N_{t}}<t$ em que elas ocorrem, são variáveis aleatórias. Além disso, pela proposição $2, N_{t}$ tem distribuição de Poisson com média

$$
\Lambda(t)=\frac{e^{\alpha}}{\beta}\left(e^{\beta t}-1\right)
$$

e, dado $N_{t}=k, k \geq 1, T_{1}<T_{2}<\ldots<T_{N_{t}}<t$ têm a distribuição conjunta das estatísticas de ordem de uma amostra casual simples de tamanho $k$ da distribuição

$$
G(y)= \begin{cases}0 & \text { se } y \leq 0 \\ \frac{\left(e^{\beta y}-1\right)}{\left(e^{\beta t}-1\right)} & \text { se } 0<y \leq t \\ 1 & \text { se } y>t\end{cases}
$$

Assim, a densidade conjunta de $N_{t}, T_{1}, T_{2}, \ldots, T_{N_{t}}$ é dada por:

$$
f\left(t_{1}, t_{2}, \ldots, t_{n}, n\right)= \begin{cases}f_{N}(n) f\left(t_{1}, t_{2}, \ldots, t_{n} \mid n\right) & \text { se } n \geq 1 \\ & \text { e } 0 \leq t_{1}<t_{2}<\ldots<t_{n}, \\ f_{N}(0) & \text { se } n=0,\end{cases}
$$

em que

$$
f_{N}(n)=\frac{\left[\frac{e^{\alpha}}{\beta}\left(e^{\beta t}-1\right)\right]^{n} e^{-\frac{e^{\alpha}}{\beta}\left(e^{\beta t}-1\right)}}{n !} \text { para } n=0,1, \ldots
$$


$\mathrm{e}$

$$
\begin{aligned}
f\left(t_{1}, t_{2}, \ldots, t_{n} \mid n\right) & =n ! \prod_{i=1}^{n} \frac{\beta e^{\beta t_{i}}}{\left(e^{\beta t}-1\right)} \\
& =n ! \frac{\beta^{n} e^{\beta \sum_{i=1}^{n} t_{i}}}{\left(e^{\beta t}-1\right)^{n}}, 0 \leq t_{1}<t_{2}<\ldots<t_{n} .
\end{aligned}
$$

Portanto,

$$
f\left(t_{1}, t_{2}, \ldots, t_{n}, n\right)= \begin{cases}e^{n \alpha-\frac{e^{\alpha}\left(e^{\beta t}-1\right)}{\beta}+\beta \sum_{i=1}^{n} t_{i}} & \text { se } n \geq 1 \\ e^{n \alpha-\frac{e^{\alpha}\left(e^{\beta t}-1\right)}{\beta}} & \text { e } 0 \leq t_{1}<t_{2}<\ldots<t_{n}\end{cases}
$$

Se $n \geq 1$, que é, obviamente, o caso de maior interesse, o logaritimo da função de verossimilhança é dado por

$$
l(\beta, \alpha ; n, \mathbf{T})=n \alpha-\frac{e^{\alpha}\left(e^{\beta t}-1\right)}{\beta}+\beta \sum_{i=1}^{n} t_{i}
$$

e os estimadores de máxima verossimilhança para os parâmetros $\beta$ e $\alpha$, são obtidos resolvendo-se o seguinte sistema não-linear de equações:

$$
\begin{aligned}
& \frac{l(\beta, \alpha ; \mathbf{X})}{\partial \beta}=-\frac{e^{\alpha}\left(e^{\beta t}(\beta t-1)+1\right)}{\beta^{2}}+\sum_{i=1}^{n} t_{i}=0 \\
& \frac{l(\beta, \alpha ; \mathbf{X})}{\partial \alpha}=n-\frac{e^{\alpha}\left(e^{\beta t}-1\right)}{\beta}=0
\end{aligned}
$$

Da equação (2.11) obtêm-se:

$$
\begin{aligned}
-\frac{e^{\alpha}\left(e^{\beta t}(\beta t-1)+1\right)}{\beta^{2}}+\sum_{i=1}^{n} t_{i}=0 & \Rightarrow e^{\alpha}\left(e^{\beta t}(\beta t-1)+1\right)=\beta^{2} \sum_{i=1}^{n} t_{i} \\
& \Rightarrow \frac{\beta^{2} \sum_{i=1}^{n} t_{i}-e^{\alpha}}{e^{\alpha}}=e^{\beta t}(\beta t-1) \\
& \Rightarrow e^{\beta t}=\frac{\beta^{2} \sum_{i=1}^{n} t_{i}-e^{\alpha}}{e^{\alpha}(\beta t-1)} \\
& \Rightarrow \beta t=\ln \left[\frac{\beta^{2} \sum_{i=1}^{n} t_{i}-e^{\alpha}}{e^{\alpha}(\beta t-1)}\right] \\
& \Rightarrow \beta=\frac{\ln \left[\frac{\beta^{2} \sum_{i=1}^{n} t_{i}-e^{\alpha}}{e^{\alpha}(\beta t-1)}\right]}{t}
\end{aligned}
$$


Substituindo o resultado acima na equação (2.12) e resolvendo em relação a $\alpha$, têm-se:

$$
\begin{aligned}
n-\frac{e^{\alpha}\left(e^{\beta t}-1\right)}{\beta}=0 & \Rightarrow e^{\alpha}\left(e^{\beta t}-1\right)=\beta n \\
& \Rightarrow e^{\alpha}=\frac{\beta n}{\left(e^{\beta t}-1\right)} \\
& \Rightarrow \alpha=\ln \left[\frac{\beta n}{\left(e^{\beta t}-1\right)}\right]
\end{aligned}
$$

Portanto, os estimadores de máxima verossimilhança para $\alpha$ e $\beta$ são dados por:

$$
\begin{gathered}
\hat{\alpha}\left(T_{1}, T_{2}, \ldots, T_{N_{t}}, N_{t}\right)=\ln \left[\frac{N_{t} \hat{\beta}\left(T_{1}, T_{2}, \ldots, T_{N_{t}}, N_{t}\right)}{\left(e^{t \hat{\beta}\left(T_{1}, T_{2}, \ldots, T_{N_{t}}, N_{t}\right)}-1\right)}\right] \\
\hat{\beta}\left(T_{1}, T_{2}, \ldots, T_{N_{t}}, N_{t}\right)=\frac{\ln \left[\frac{\widehat{\beta}^{2}\left(T_{1}, T_{2}, \ldots, T_{N_{t}}, N_{t}\right) \sum_{i=1}^{N_{t}} t_{i}-e^{\hat{\alpha}\left(T_{1}, T_{2}, \ldots, T_{N_{t}}, N_{t}\right)}}{e^{\dot{\alpha}\left(T_{1}, T_{2}, \ldots, T_{N_{t}}, N_{t}\right)}\left(t \hat{\beta}\left(T_{1}, T_{2}, \ldots, T_{N_{t}}, N_{t}\right)-1\right)}\right]}{t}
\end{gathered}
$$

A solução do sistema não-linear de equações acima, para a obtenção das estimativas de $\alpha$ e $\beta$ depende da aplicação de métodos interativos.

\subsubsection{Dados com Censura Tipo II}

Sob censura tipo II, o sistema é observado até a ocorrência da n-ésima falha, isto é, até o instante $T_{n}$. Neste caso, segue da proposição 2 que

$$
T_{1}<T_{2}<\ldots<T_{n}
$$

têm densidade conjunta dada por

$$
\begin{aligned}
f\left(t_{1}, t_{2}, \ldots, t_{n}\right) & =\prod_{i=1}^{n} \lambda\left(t_{i}\right) e^{-\int_{0}^{t_{n}} \lambda(x) d x} \\
& =e^{\left(n \alpha+\beta \sum_{i=1}^{n} t_{i}\right)} e^{-\frac{e^{\alpha}}{\beta}\left(e^{\left(3 t_{n}\right)}-1\right)},
\end{aligned}
$$

$0 \leq t_{1}<t_{2}<\ldots<t_{n}$ 
O Logaritimo da função de verossimilhança, nesse caso, é dado por:

$$
l(\beta, \alpha ; \mathbf{T})=n \alpha+\beta \sum_{i=1}^{n} t_{i}-\frac{e^{\alpha}}{\beta}\left(e^{\left(\beta t_{n}\right)}-1\right)
$$

e os estimadores de máxima verossimilhança para os parâmetros $\alpha$ e $\beta$ são obtidos resolvendo-se o seguinte sistema não-linear de equações:

$$
\begin{aligned}
& \frac{l(\beta, \alpha ; \mathbf{X})}{\partial \alpha}=n-\frac{e^{\alpha}}{\beta}\left(e^{\left(\beta t_{n}\right)}-1\right)=0 \\
& \frac{l(\beta, \alpha ; \mathbf{X})}{\partial \beta}=\sum_{i=1}^{n} t_{i}-\frac{e^{\alpha}}{\beta^{2}}-\frac{e^{\alpha}}{\beta^{2}}\left(e^{\left(\beta t_{n}\right)}-1\right)\left(\beta t_{n}-1\right)=0
\end{aligned}
$$

Da equação (2.15) têm-se:

$$
\begin{aligned}
\beta n-e^{\alpha}\left(e^{\left(\beta t_{n}\right)}-1\right)=0 & \Rightarrow \beta n=e^{\alpha}\left(e^{\left(\beta t_{n}\right)}-1\right) \\
& \Rightarrow e^{\alpha}=\frac{\beta n}{\left(e^{\left(\beta t_{n}\right)}-1\right)} \\
& \Rightarrow \alpha=\ln \left(\frac{\beta n}{\left(e^{\left(\beta t_{n}\right)}-1\right)}\right)
\end{aligned}
$$

Substituindo o resultado acima na equação (2.16) e resolvendo em relação a $\beta$, tem-se

$$
\begin{aligned}
0=\beta^{2} \sum_{i=1}^{n} t_{i}-e^{\alpha}-e^{\alpha} e^{\beta t_{n}}\left(\beta t_{n}-1\right) & \Rightarrow \beta^{2} \sum_{i=1}^{n} t_{i}=e^{\alpha}+e^{\alpha} e^{\beta t_{n}}\left(\beta t_{n}-1\right) \\
& \Rightarrow \frac{\beta^{2} \sum_{i=1}^{n} t_{i}}{e^{\alpha}}=1+e^{\beta t_{n}}\left(\beta t_{n}-1\right) \\
& \Rightarrow \frac{\beta^{2} \sum_{i=1}^{n} t_{i}-e^{\alpha}}{e^{\alpha}}=e^{\beta t_{n}}\left(\beta t_{n}-1\right) \\
& \Rightarrow e^{\beta t_{n}}=\frac{\beta^{2} \sum_{i=1}^{n} t_{i}-e^{\alpha}}{\left(\beta t_{n}-1\right) e^{\alpha}} \\
& \Rightarrow \beta=\frac{1}{t_{n}} \ln \left(\frac{\beta^{2} \sum_{i=1}^{n} t_{i}-e^{\alpha}}{\left(\beta t_{n}-1\right) e^{\alpha}}\right)
\end{aligned}
$$

Portanto, os etimadores de máxima verossimilhança para $\alpha$ e $\beta$ são dados por:

$$
\begin{gathered}
\hat{\alpha}\left(T_{1}, T_{2}, \ldots, T_{n}\right)=\ln \left(\frac{n \hat{\beta}\left(T_{1}, T_{2}, \ldots, T_{n}\right)}{e^{t_{n} \hat{\beta}\left(T_{1}, T_{2}, \ldots, T_{n}\right)}-1}\right) \\
\hat{\beta}\left(T_{1}, T_{2}, \ldots, T_{n}\right)=\frac{1}{t_{n}} \ln \left(\frac{\hat{\beta}^{2}\left(T_{1}, T_{2}, \ldots, T_{n}\right) \sum_{i=1}^{n} t_{i}-e^{\hat{\alpha}\left(T_{1}, T_{2}, \ldots, T_{n}\right)}}{\left(t_{n} \hat{\beta}\left(T_{1}, T_{2}, \ldots, T_{n}\right)-1\right) e^{\hat{\alpha}\left(T_{1}, T_{2}, \ldots, T_{n}\right)}}\right)
\end{gathered}
$$


A solução do sistema não-linear de equações acima, para a obtenção das estimativas de $\alpha$ e $\beta$ depende da aplicação de métodos interativos.

\subsection{Modelo Misto sob Censura Tipo II}

Sob o modelo misto, os instantes de falha experimentados pelo sistema se comportam como o processo de chegadas, $\left\{T_{n}: n \geq 1\right\}$, de um processo pontual de Poisson, $\left\{N_{t}: t \geq 0\right\}$, com função intensidade completa dada por

$$
\lambda\left(t \mid N_{u}: u<t\right)=\exp \left\{\Theta^{T} \mathbf{Z}\left(t \mid N_{u}: u<t\right)\right\},
$$

em que $\Theta^{T}=\left(\theta_{1}, \ldots, \theta_{k}\right)$ é um vetor de parâmetros e

$$
\mathbf{Z}^{T}\left(t \mid N_{u}: u<t\right)=\left(Z_{1}\left(t \mid N_{u}: u<t\right), \ldots, Z_{k}\left(t \mid N_{u}: u<t\right)\right)
$$

um vetor de funções intensidades completas.

Assim, se o sistema é observado até a ocorrência da n-ésima falha, isto é, até o instante $T_{n}$, segue da proposição 2 que

$$
T_{1}<T_{2}<\ldots<T_{n}
$$

têm densidade conjunta dada por

$$
f\left(t_{1}, t_{2}, \ldots, t_{n}\right)=\prod_{i=1}^{n} \exp \left\{\Theta^{T} \mathbf{Z}\left(t_{i}\right)\right\} \exp \left\{-\int_{0}^{t_{n}} \exp \left\{\Theta^{T} \mathbf{Z}(t)\right\} d t\right\},
$$

$0 \leq t_{1}<t_{2}<\ldots<t_{n}$, em que, por simplicidade, $\mathbf{Z}(t)=\mathbf{Z}\left(t \mid N_{u}: u<t\right)$.

O logaritimo da função de verossimilhança, nesse caso, é dado por:

$$
l(\Theta)=\sum_{i=1}^{n} \Theta^{T} \mathbf{Z}\left(t_{i}\right)-\int_{0}^{t_{n}} \exp \left\{\Theta^{T} \mathbf{Z}(t)\right\} d t
$$

e o estimador de máxima verossimilhança de $\Theta$ é obtido resolvendo-se o seguinte sistema não-linear de equações

$$
U_{r}(\Theta)=0
$$


$r=1, \ldots, p$, em que $U_{r}, r=1, \ldots, p$, são as funções de score associadas a $r$.

Mais precisamente,

$$
U_{r}(\Theta)=\frac{\partial l(\Theta)}{\partial \theta_{r}}
$$

$r=1, \ldots, p$.

Observando que

$$
\Theta=\left(\begin{array}{c}
\theta_{1} \\
\theta_{2} \\
\vdots \\
\theta_{r-1} \\
\theta_{r} \\
\theta_{r+1} \\
\vdots \\
\theta_{p}
\end{array}\right) \Rightarrow \frac{\partial \Theta}{\theta_{r}}=\left(\begin{array}{c}
0 \\
0 \\
\vdots \\
0 \\
1 \\
0 \\
\vdots \\
0
\end{array}\right)
$$

e que

$$
\left(\frac{\partial \Theta}{\partial \theta_{r}}\right)^{T} \mathbf{Z}\left(t_{i}\right)=Z_{r}\left(t_{i}\right)
$$

têm-se

$$
U_{r}(\Theta)=\sum_{i=1}^{n} Z_{r}\left(t_{i}\right)-\int_{0}^{t_{n}} Z_{r}(t) \exp \left\{\Theta^{T} \mathbf{Z}(t)\right\} d t
$$

$r=1, \ldots, p$.

Assim, a entrada $(r, s), 1 \leq r, s \leq p$, da matriz $p \times p$ de informação observada é dada por

$$
I_{r s}(\Theta)=\frac{-\partial^{2} l(\Theta)}{\partial \theta_{r} \partial \theta_{s}}=\int_{0}^{t_{n}} Z_{r}(t) Z_{s}(t) \exp \left\{\Theta^{T} \mathbf{Z}(t)\right\} d t .
$$

Os valores das integrais acima, entretanto, dificilmente podem ser obtidos analiticamente. De um modo geral, a utilização de técnicas de integração numérica é necessária. Da mesma forma, a estimativa de máxima verossimilhança, $\hat{\Theta}$, de $\Theta$, é geralmente obtida através da utilização do método iterativo de Newton-Raphson. A fórmula de interação é dada por: 


$$
\Theta^{(j+1)}=\Theta^{(j)}+I\left(\Theta^{(j)}\right) U\left(\Theta^{(j)}\right) \text { para } j=1,2, \ldots,
$$

em que $\Theta^{(1)}$ é uma aproximação inicial para $\hat{\Theta}$. A convergência ocorre quando:

- $\Theta^{(i+1)}$ estiver próximo de $\Theta^{(i)}$,

- $I\left(\Theta^{(i+1)}\right)$ estiver próximo de $I\left(\Theta^{(i)}\right)$.

Para calcularmos $l(\Theta), U(\Theta)$ ou $I(\Theta)$, é necessário ainda utilizar métodos de integração numérica. Esses métodos consistem em determinar $0=a_{1}<$ $a_{2}<\ldots<a_{m}=t_{n}$ e constantes $w_{1}, w_{2}, \ldots, w_{m}$, tais que

$$
\sum_{j=1}^{m} w_{j} \exp \left(\Theta^{T} \mathbf{Z}\left(a_{j}\right)\right) \cong \int_{0}^{t_{n}} \exp \left(\Theta^{T} \mathbf{Z}(t)\right) d t .
$$

Como a trajetória de $\mathbf{Z}(t)$ apresenta descontinuidades nos instantes $t_{1}, \ldots, t_{n}$, é conveniente aplicar esses métodos a cada parcela da soma abaixo

$$
\int_{0}^{t_{n}} \exp \left(\Theta^{T} \mathbf{Z}(t)\right) d t=\sum_{i=1}^{n} \int_{t_{i-1}}^{t_{i}} \exp \left(\Theta^{T} \mathbf{Z}(t)\right) d t .
$$

A determinação das constantes $w_{i 1}, w_{i 2}, \ldots, w_{i m}$ e $t_{i-1}=a_{i 1}<a_{i 2}<\ldots<$ $a_{i m}=t_{i}$, tais que

$$
\sum_{j=1}^{m} w_{i j} \exp \left(\Theta^{T} \mathbf{Z}\left(a_{i j}\right)\right) \cong \int_{t_{i-1}}^{t_{i}} \exp \left(\Theta^{T} \mathbf{Z}(t)\right) d t
$$

são feita através de uma regra de quadratura. As regras de quadratura podem ser construídas com o auxílio de softwares de integração numérica ou mesma com a utilização de um método simples como, por exemplo, a regra do trapezóide de Simpson, aproximando assim a i-ésima integral em (2.26).

A regra do trapezóide se Simpson consiste, basicamente, das seguintes etapas:

1. Selecionar um inteiro positivo $k_{i}$ e definir

$$
\Delta_{i}=\frac{\left(t_{i}-t_{i-1}\right)}{2 k_{i}}
$$


2. Definir os $a_{i^{\prime} s}$ por

$$
a_{i j}=t_{i-1}+(j-1) \Delta_{i}
$$

para $j=1, \ldots, 2 k_{i}+1$.

3. Definir os $w_{i^{\prime} s}$ por

$$
\begin{aligned}
& w_{i 1}=\ldots=w_{i 2 k_{i}+1}=\frac{1}{3} \\
& w_{i 2}=\ldots=w_{i 2 k_{i}}=\frac{4}{3} \\
& w_{i 3}=\ldots=w_{i 2 k_{i}-1}=\frac{2}{3}
\end{aligned}
$$

4. Fazer

$$
\int_{t_{i-1}}^{t_{i}} \exp \left(\Theta^{T} \mathbf{Z}(t)\right) d t=\sum_{j=1}^{2 k_{i}+1} w_{i j} \exp \left(\Theta^{T} \mathbf{Z}\left(a_{i j}\right)\right)
$$

Desta forma, utilizando as equações (2.21 - 2.23), podemos aproximar as equações $l(\Theta), U(\Theta)$ e $I(\Theta)$ pelas seguintes expressões:

$$
\begin{aligned}
l^{A}(\Theta) & =\sum_{i=1}^{n} \Theta^{T} \mathbf{Z}\left(t_{i}\right)-\sum_{j=1}^{m} w_{j} \exp \left(\Theta^{T} \mathbf{Z}\left(a_{j}\right)\right) \\
\mathbf{U}_{\mathbf{r}}^{\mathbf{A}}(\theta) & =\sum_{i=1}^{n} \mathbf{Z}_{\mathbf{r}}\left(t_{i}\right)-\sum_{j=1}^{m} w_{j} \mathbf{Z}_{\mathbf{r}}\left(a_{j}\right) \exp \left(\Theta^{T} \mathbf{Z}\left(a_{j}\right)\right) \\
\mathbf{I}_{\mathbf{r} \mathbf{s}}^{\mathbf{A}}(\theta) & =\sum_{j=1}^{m} w_{j} \mathbf{Z}_{\mathbf{r}}\left(a_{j}\right) \mathbf{Z}_{\mathbf{s}}\left(a_{j}\right) \exp \left(\Theta^{T} \mathbf{Z}\left(a_{j}\right)\right)
\end{aligned}
$$

Outros métodos de integração numérica e/ou outras regras de quadratura podem ser encontradas em [32].

\subsubsection{Teste de Hipótese}

Como vimos no Capítulo $1, \mathbf{Z}^{T}\left(t \mid N_{u}: u<t\right)$, no modelo misto, é um vetor de funções intensidades completas. Em geral desenhamos $\mathbf{Z}^{T}\left(t \mid N_{u}: u<t\right)$ de tal forma que as componentes deste vetor expressem funções de intensidades 
com padrões de renovação e de tendência temporal. Entretanto o processo pontual nem sempre apresenta essas duas características.

Assim sendo, faz-se necessário verificar a signifcância de cada um dos parâmetros $\theta_{i}$, associados às funções de $\mathbf{Z}^{T}\left(t \mid N_{u}: u<t\right)$ que incorporam as características específicas de interesse, e determinar se os dados suportam a incorporação destes componentes no modelo.

Dentre os diversos testes que encontramos na literaturas, iremos utilizar neste trabalho apenas os testes de Wald e da da Razão de Verossimilhanças. Outros testes podem ser encontrados em [8].

\section{Teste de Wald}

O teste de Wald baseia-se na estatística:

$$
\frac{\left(\hat{\theta}_{i}-\theta_{i\left(H_{0}\right)}\right)^{2}}{I_{i i}^{-1}(\hat{\Theta})} \sim \chi_{1}^{2},
$$

em que $\hat{\theta}_{i}$ é o estimador de máxima verossimilhança da i-ésima componente do vetor $\Theta, \theta_{i\left(H_{0}\right)}$, é o valor desta componente, sob a hipótese $H_{0}$ a ser testada e $I_{i i}^{-1}(\hat{\Theta})$ é a entrada $(i, i)$ da inversa da matriz de informação, $I(\boldsymbol{\Theta})$, calculada no ponto $\Theta=\hat{\Theta}$.

Nos estudos de confiabilidade, entretanto, costuma-se testar apenas as hipóteses $H_{0}: \theta_{i}=0$. Neste caso, o teste baseia-se, simplesmente, na aproximação

$$
\frac{\hat{\theta}_{i}}{\left[I_{i i}^{-1}(\hat{\mathbf{\Theta}})\right]^{1 / 2}} \sim N(0,1) .
$$

Portanto, ao nível de significância $\alpha$, o teste que rejeita $H_{0}$, se e somente se, 


$$
\left|\frac{\hat{\theta}_{i}}{\left[I_{i i}^{-1}(\hat{\Theta})\right]^{1 / 2}}\right|>Z_{1-\alpha / 2}
$$

Se a hipótese $H_{0}$ : $\theta_{i}=0$ é rejeitada, dizemos que o parâmetro $\theta_{i}$ é significante.

\section{Teste de Razão de Verossimilhanças}

Já o teste da Razão de Verossimilhanças baseia-se na estatística

$$
R=2 l(\hat{\Theta})-2 l(\tilde{\Theta}), \operatorname{com} R \sim \chi_{k}^{2}
$$

em que $\hat{\Theta}$ é o vetor de parâmetros estimados do modelo, $\tilde{\Theta}$ é o vetor de parâmetros estimados do modelo sob $H_{0}$ e $k$ o número de parâmetros restritos sob $H_{0}$. No caso da análise da confiabilidade, o interesse é testar $k$ parâmetros $\theta_{i}$ do vetor $\hat{\Theta}$ iguais a zero.

Em geral, testa-se apenas 1 parâmetro por vez. Nestes casos, basta comparar o valor de $2 l(\hat{\Theta})-2 l(\tilde{\Theta})$ com o percentil de ordem $(1-\alpha)$ de uma distribuição Qui-quadrado com 1 grau de liberdade. Rejeita-se a hipótese $H_{0}$ se o valor observado da estatística for superior ao valor do percentil $(1-\alpha)$ da distribuição Qui-quadrado com 1 grau de liberdade, onde $\alpha$ é o nível de significância.

\subsubsection{Análise do Ajuste do Modelo}

Após testar a significância dos parâmetros do modelo e determinar o modelo parcimonioso, é conveniente verificar a qualidade do seu ajuste. No tipo de modelagem que estamos trabalhando, essa análise é feita com base nos resíduos generalizados, isto é

$$
\hat{e}_{i}=\int_{t_{i-1}}^{t_{i}} \hat{\lambda}\left(s \mid N_{u}: u<s\right) d s=\hat{\Lambda}\left(t_{i}\right)-\hat{\Lambda}\left(t_{i-1}\right)
$$


em que $\int_{0}^{t} \lambda\left(s \mid N_{u}: u<s\right) d s=\Lambda(t)$

Se o modelo estiver bem ajustado, os resíduos, $\hat{e}_{i^{\prime} s}$, devem comportar-se, aproximadamente, como variáveis aleatórias independentes, identicamente distribuídas, com distribuição exponencial padrão. Para verificar se os $\hat{e}_{i^{\prime} s}$ exibem este comportamento, podemos utilizar tanto os testes de hipótese baseados no conceito de Total Time on Test (TTT), quanto técnicas gráficas heurísticas tais como o gráfico de Probabilidades Exponencial ou o gráfico Quantil-Quantil.

Um cuidado que se deve tomar é quando existe a presença de clusters de eventos, ou seja, quando vários eventos são reportados em instantes muito próximos. Isto pode ocorrer devido a deficiências tanto no processo de coleta de dados quanto no de reparo do sistema. Nesses casos, o modelo poderá não se ajustar bem ao conjunto de dados.

\subsubsection{Multiplas Unidades Amostrais}

Nas seções anteriores tratamos a questão inferencial apenas no caso em que uma única unidade amostral ${ }^{1}$ é observada. A literatura sobre a análise da confiabilidade de sistemas tem focado seus trabalhos principalmente neste caso pois grande parte do desenvolvimento dessas técnicas foram feitas pelo setor nuclear e aero-espacial, onde o custo de uma unidade amostral é muito alto. Assim sendo, as análises estão focadas na obtenção de informações específicas sobre a unidade amostral em teste, e não para realizar inferências sobre uma população delas.

Entretanto, este tipo de análise é útil também em situações nas quais desejamos estender os resultados da análise estatística para uma população de interesse. Nesses casos, a utilização de uma única unidade amostral não é adequada.

Para utilizarmos diversas unidades amostrais, devemos entender a simila-

\footnotetext{
${ }^{1}$ Produto ou Sistema
} 
riedade delas tanto em relação a sua composição física, ao ambiente e nível de estresse que estas estão expostas. Estaremos abordando nesta seção apenas o caso em que as unidades amostrais são idênticas e estão sujeitas as mesmas condições ambientais e de utilização.

Para este caso a função de verossimilhança do modelo é dada por:

$$
L(\theta)=\prod_{i=1}^{k}\left(\prod_{j=1}^{n_{i}} \lambda\left(t_{i j} \mid N_{u}: u<s\right)\right) e^{-\int_{0}^{t_{n_{i}}} \lambda\left(s \mid N_{u}: u<s\right) d s}
$$

onde $t_{i j}$ é a j-ésima falha da i-ésima unidade amostral, com $i=1,2, \ldots, k$ e $j=1, \ldots, n_{i}$. No entanto, utilizando a função intensidade descrita em (2.19), a verossimilhança pode ser reescrita como:

$$
L(\theta)=\prod_{i=1}^{k} \prod_{j=1}^{n_{i}} \exp \left\{\Theta^{T} \mathbf{Z}\left(t_{i j}\right)\right\} \exp \left\{-\int_{0}^{t_{n_{i}}} \exp \left\{\Theta^{T} \mathbf{Z}\left(t_{i}\right)\right\} d t\right\}
$$

Da mesma maneira que obtivemos as estimativas de máxima verossimilhança para o caso de uma única unidade amostral, devemos utilizar estes mesmos princípios para o caso de múltiplas unidades amostrais. Ressaltamos que a maximização da função de verossimilhança acima pelos métodos numéricos convencionais, em geral apresentam bons resultados, entretanto a convergência do método iterativo depende fortemente do vetor de parâmetro que irá iniciar o processo e, não existe nenhum critério ótimo para uma escolha adequada desse inicializador. Neste trabalho utilizamos como vetor de inicialização para o processo iterativo a estimativa de verossimilhança do parâmetro $\alpha^{2}$ além de fixar, inicialmente, $\beta=\gamma=0$.

Os testes de hipóteses para testar a significância dos parâmetros do modelo são idênticos ao caso de uma única unidade amostral. Já para a análise de resíduos, como estamos trabalhando com múltiplas unidades amostrais, o resíduo generalizado fica dado por:

\footnotetext{
${ }^{2}$ Considerando um processo de Poisson Homogêneo, ou seja $\lambda\left(t_{i j} \mid N_{u}: u<s\right)=e^{\alpha}$
} 


$$
\hat{e}_{i j}=\int_{t_{(i-1) j}}^{t_{i j}} \hat{\lambda}\left(s \mid N_{u}: u<s\right) d s=\hat{\Lambda}\left(t_{i j}\right)-\hat{\Lambda}\left(t_{(i-1) j}\right)
$$

$\operatorname{com} i=1, \ldots, k, j=1, \ldots, n_{i}$ e $\int_{0}^{t} \lambda\left(s \mid N_{u}: u<s\right) d s=\Lambda(t)$.

Se o modelo estiver bem ajustado, os resíduos, $\hat{e}_{i j^{\prime} s}$, devem comportar-se, aproximadamente, como variáveis aleatórias independentes, identicamente distribuídas, com distribuição exponencial padrão. Para verificar se os $\hat{e}_{i^{\prime} s}$ exibem este comportamento, podemos utilizar tanto os testes de hipótese baseados no conceito de Total Time on Test (TTT), quanto técnicas gráficas heurísticas tais como o gráfico de Probabilidades Exponencial ou o gráfico Quantil-Quantil (QQ-Plot).

Um cuidado que se deve tomar é quando existe a presença de clusters de eventos, ou seja, quando vários eventos são reportados em instantes muito próximos. Isto pode ocorrer devido a deficiências tanto no processo de coleta de dados quanto no de reparo do sistema. Nesses casos, o modelo poderá não se ajustar bem ao conjunto de dados. É recomendado que gráficos tipo Quantil-Quantil sejam feitos separadamente para cada uma das unidades amostrais com o objetivo de verificar uma possível inadequabilidade do modelo para uma determinada unidade amostral. 


\section{CAPÍTULO 3}

\section{Aplicações}

Nos capítulos anteriores, apresentamos todo o ferramental metodológico para a aplicação de processos pontuais mistos à análise da confiabilidade de sistemas reparáveis. Neste capítulo iremos aplicar esse ferramental em casos reais. Iremos analisar dois conjuntos de dados provenientes de estudos com uma única unidade amostral e um outro proveniente de um estudo com várias unidades amostrais.

\subsection{Estudo de Caso I}

Neste estudo de caso vamos utilizar dados de falhas do motor principal de propursão do submarino USS Grampus, da marinha americana. Esses dados são reais e comumente utilizados em livros que tratam da análise de dados recorrentes.

Como modelo de base para analise, utilizaremos o modelo de processo pontual com função intensidade completa dada por

$$
\lambda\left(t \mid N_{u}: u<t\right)=\exp \left\{\Theta^{T} \mathbf{Z}\left(t \mid N_{u}: u<t\right)\right\}
$$

com

$$
\Theta=\left(\begin{array}{c}
\alpha \\
\beta \\
\gamma
\end{array}\right), \mathbf{Z}(t)=\left(\begin{array}{c}
1 \\
t \\
t_{N_{t-}}
\end{array}\right)
$$

em que $t_{N_{t-}}$ é o tempo decorrido desde a última falha do sistema. 
Os tempos, em milhares de horas, das paradas não planejadas do USS Grampus, encontram-se no quadro abaixo.

\begin{tabular}{|ccccccccc|}
\hline 860 & 2122 & 4000 & 4910 & 6613 & 9042 & 11511 & 12795 & 14028 \\
1258 & 2439 & 4247 & 5676 & 6975 & 9330 & 11575 & 13399 & 14035 \\
1317 & 3203 & 4411 & 5755 & 7335 & 9394 & 12100 & 13668 & 14173 \\
1442 & 3298 & 4456 & 6137 & 8158 & 9426 & 12126 & 13780 & 14449 \\
1897 & 3902 & 4517 & 6221 & 8498 & 9872 & 12368 & 13877 & 14587 \\
2011 & 3910 & 4899 & 6311 & 8690 & 10191 & 12681 & 14007 & 14610 \\
15070 & & & & & & & & \\
\hline
\end{tabular}

Baseado no conjunto de dados acima, as estimativas para os parâmetros da função intensidade de falha completa, encontram-se abaixo.

\begin{tabular}{ccccc}
\hline \hline Parâmetro & Estimativa & Erro Padrão & Estatística de Wald & P-Value \\
\hline \hline$\widehat{\alpha}$ & -5.913 & 0.321 & 340.10 & $<0.01$ \\
$\widehat{\beta}$ & 0.0000312 & 0.000031 & 1.05 & 0.31 \\
$\widehat{\gamma}$ & 0.0001853 & 0.000521 & 0.13 & 0.72 \\
\hline \hline
\end{tabular}

Com base nas estimativas acima, o valor da função de verossimilhança é de $l(\theta)=-363.1$. Podemos também verificar que o teste de Wald não rejeita a hipótese $H_{0}: \gamma=0$, a níveis de significância usuais. Pode-se também verificar que o teste de Wald também não rejeita a hipótese $H_{0}: \beta=0$, a níveis de significâncias usuais. Antes de fazer qualquer inferência sobre $\beta$, iremos recalcular as estimativas do modelo, considerando a hipótese $H_{0}: \gamma=0$. As novas estimativas, encontram-se na tabela abaixo.

\begin{tabular}{ccccc}
\hline \hline Parâmetro & Estimativa & Erro Padrão & Estatística de Wald & P-Value \\
\hline \hline$\widehat{\alpha}$ & $-\overline{5} .864$ & 0.2873 & 416.60 & $<0.01$ \\
$\widehat{\beta}$ & 0.000032 & 0.00003177 & 1.05 & 0.31 \\
\hline \hline
\end{tabular}

Com base nas estimativas acima, o valor da função de verossimilhança é de $l(\theta)=-363.2$. Sendo assim o valor observado da estatística do teste da 
Razão de Verossimilhanças, que é dado por $2 l(\widehat{\theta})-2 l(\widetilde{\theta})=0.2$, confirma a não rejeição do teste de Wald para $H_{0}: \gamma=0$. Em termos práticos, a não rejeição de $H_{0}$ indica que, em princípio, as falhas experimentadas pelo motor de propulsão do USS Grampus, não apresentam um padrão de renovação.

Mais uma vez, utilizando o teste de Wald, agora para $H_{0}: \beta=0$, podemos verificar que o $\mathrm{P}$-value do teste é igual a 0.31 , sinalizando para a não rejeição da hipótese $H_{0}: \beta=0$. Em termos práticos isto indica que, em princípio, as falhas experimentadas pelo motor de propulsão do USS Grampus, não apresentam um comportamento de tendência (crescente ou decrescente) ao longo do tempo.

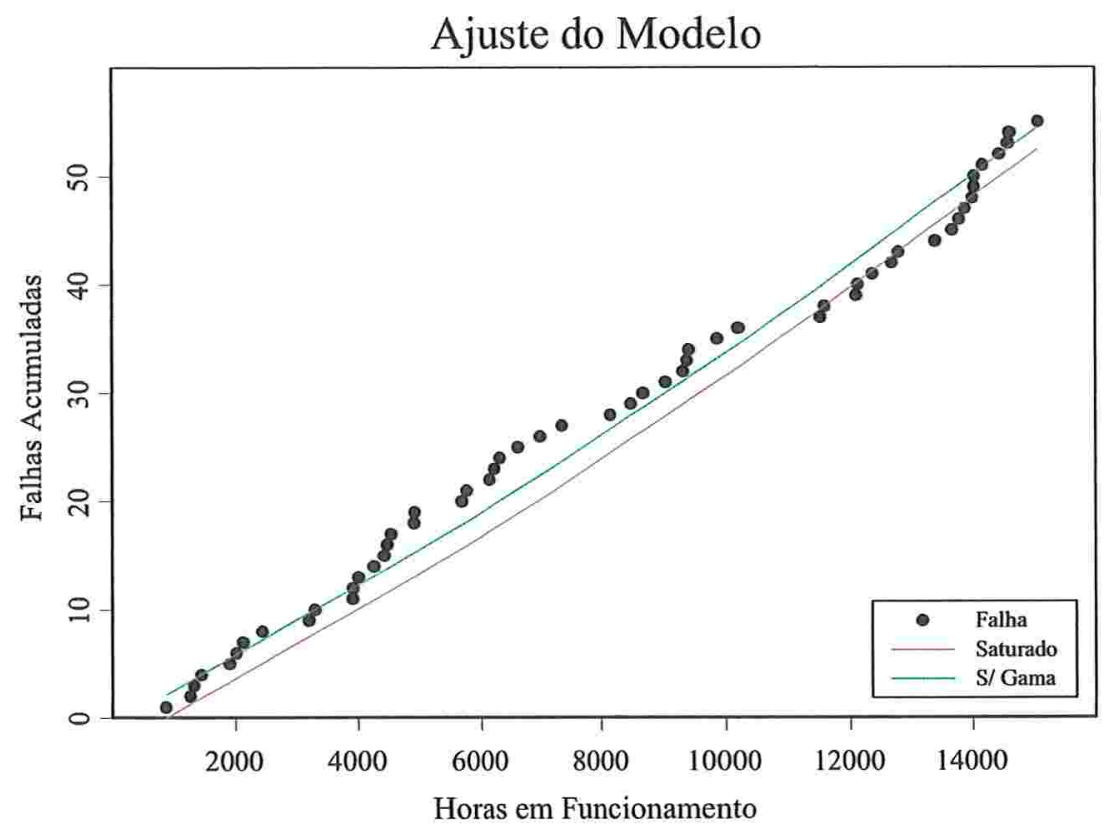

Figura 3.1: Comparação de Ajustes - USS Grampus

Podemos verificar no gráfico da Figura 3.1 que o modelo saturado ${ }^{1}$ pouco difere-se do modelo sem o parâmetro $\gamma$. Isto realmente mostra que a exclusão do parâmetro $\gamma$ pouco afeta o ajuste do modelo.

\footnotetext{
${ }^{1}$ com o parâmetro $\gamma$
} 
Por fim o modelo que nos resta é o modelo reduzido. No modelo reduzido, estamos considerando que a taxa de falha é constante com o decorrer do tempo, ou seja, trata-se de um processo de Poison homogêneo. Para este modelo, a estimativa do parâmetro encontra-se abaixo.

\begin{tabular}{ccccc}
\hline \hline Parâmetro & Estimativa & Erro Padrão & Estatística de Wald & P-Value \\
\hline \hline$\widehat{\alpha}$ & $-\check{0} .613$ & 0.1348 & 1734 & $<0.01$ \\
\hline \hline
\end{tabular}

O valor da função de verossimilhança, com base na estimativas acima foi de $l(\theta)=-363.7$. Sendo assim o valor da estatística do teste da Razão de Verossimilhanças é $2 l(\widehat{\theta})-2 l(\widetilde{\theta}) \approx 1.0$, o que aponta para a não rejeição da hipótese $H_{0}: \beta=0$, conforme o teste de Wald já havia indicado. A Figura 3.2 corresponde ao gráfico do modelo ajustado (Reduzido).

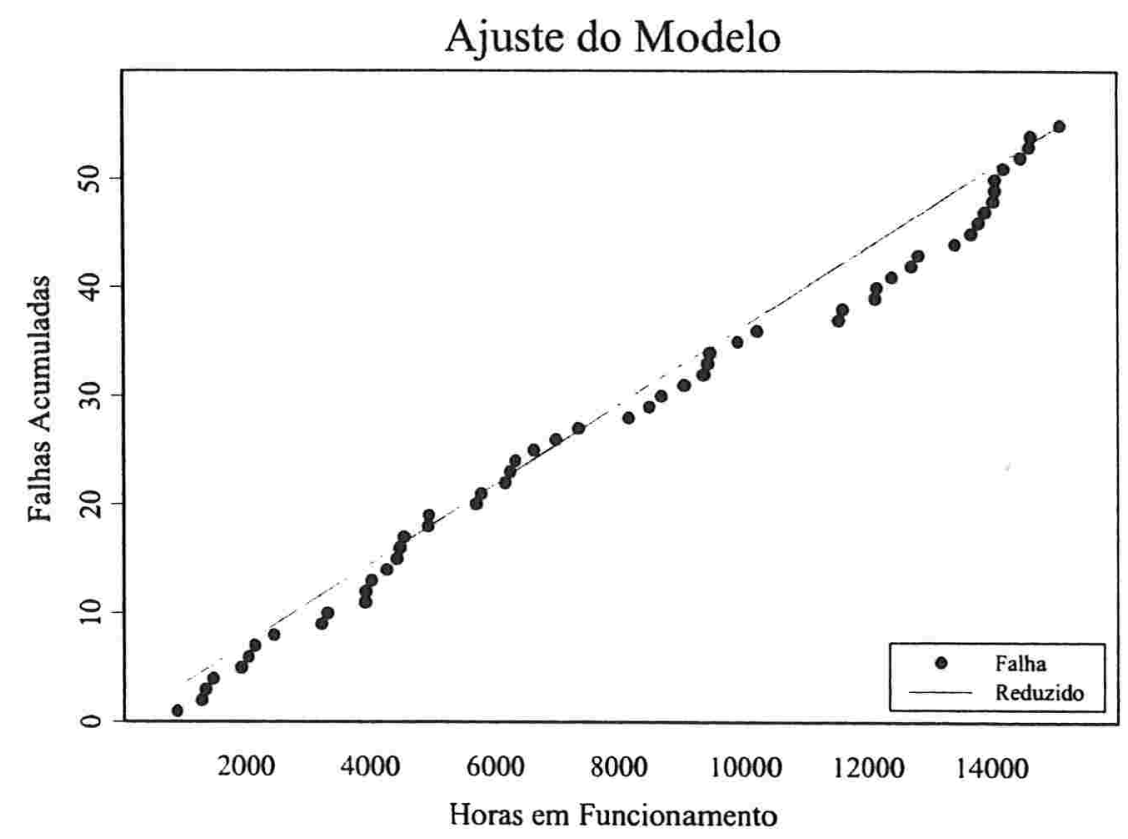

Figura 3.2: Modelo Ajustado - LSS Grampus

Assim, podemos verificar que o comportamento das falhas do motor principal do LSS Grampus segue um processo de Poison homogêneo, e que o 
tempo entre sucessivas falhas tem distribuição exponencial.

Para verificar a qualidade do ajuste do modelo, iremos fazer uma análise dos resíduos. Utilizaremos como ferramenta um gráfico quantil-quantil dos resíduos generalizados.

\section{Grafico Quantil-Quantil (QQ Plot)}

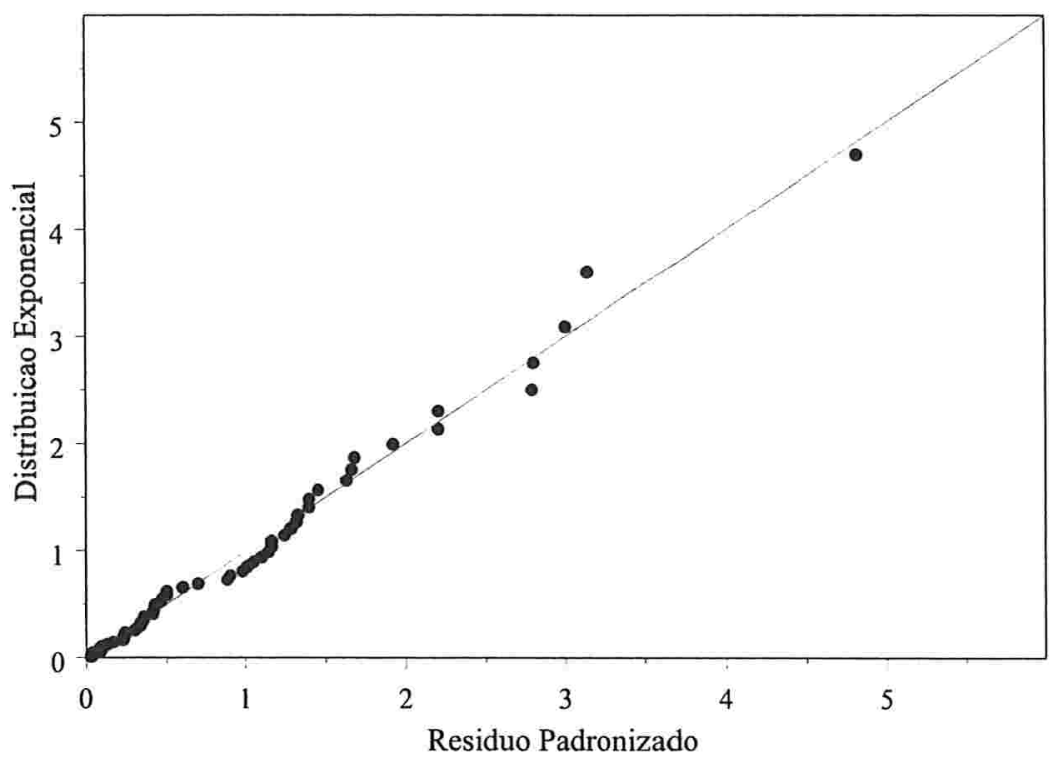

Figura 3.3: QQ Plot dos Resíduos - USS Grampus

De fato, o QQ-Plot da Figura 3.3 indica que os resíduos seguem uma distribuição exponencial padrão. Consequentemente, podemos considerar que o modelo reduzido está bem ajustado.

\subsection{Estudo de Caso II}

Neste estudo de caso vamos utilizar dados de falhas do motor principal de propursão do navio USS Halfbeak.

Como modelo de base para analise, utilizaremos o modelo de processo 
pontual com função intensidade completa dada por

$$
\lambda\left(t \mid N_{u}: u<t\right)=\exp \left\{\Theta^{T} \mathbf{Z}\left(t \mid N_{u}: u<t\right)\right\}
$$

com

$$
\Theta=\left(\begin{array}{c}
\alpha \\
\beta \\
\gamma
\end{array}\right), \mathbf{Z}(t)=\left(\begin{array}{c}
1 \\
t \\
t_{N_{t-}}
\end{array}\right)
$$

em que $t_{N_{t-}}$ é o tempo decorrido desde a última falha do sistema.

Os tempos, em milhares de horas, das paradas não planejadas do USS Halfbeak, encontram-se no quadro abaixo.

\begin{tabular}{|cccccccccc|}
\hline 1382 & 9794 & 17632 & 19940 & 21061 & 21603 & 21888 & 22635 & 23491 & 25000 \\
2990 & 10848 & 18122 & 19944 & 21309 & 21658 & 21930 & 22669 & 23526 & 25010 \\
4124 & 11993 & 19067 & 20121 & 21310 & 21688 & 21943 & 22691 & 23774 & 25048 \\
6827 & 12300 & 19172 & 20132 & 21378 & 21750 & 21946 & 22846 & 23791 & 25268 \\
7472 & 15413 & 19299 & 20431 & 21391 & 21815 & 22181 & 22947 & 23822 & 25400 \\
7567 & 16497 & 19360 & 20525 & 21456 & 21820 & 22311 & 23149 & 24006 & 25500 \\
8845 & 17352 & 19686 & 21057 & 21461 & 21822 & 22634 & 23305 & 24286 & 25518 \\
9450 & & & & & & & & & \\
\hline
\end{tabular}

Baseado no conjunto de dados acima, as estimativas para os parâmetros da função intensidade de falha completa, encontram-se abaixo.

\begin{tabular}{ccccc}
\hline \hline Parâmetro & Estimativa & Erro Padrão & Estatística de Wald & P-Value \\
\hline \hline$\widehat{\alpha}$ & -8.188 & 0.5368 & 15.25 & $<0.010$ \\
$\widehat{\beta}$ & 0.000144 & 0.00002446 & 5.886 & 0.015 \\
$\widehat{\gamma}$ & -0.0001146 & 0.0002547 & 0.4501 & 0.5023 \\
\hline \hline
\end{tabular}

Podemos verificar na tabela acima, que o teste de Wald não rejeita a hipótese $H_{0}: \gamma=0$, a níveis de significância usuais. Em termos práticos, 
isto significa que as falhas experimentadas pelo motor de propulsão do USS Halfbeak, não apresentam um padrão de renovação. Isto posto, iremos calcular as estimativas do modelo levando este fato em consideração. As novas estimativas, encontram-se na tabela abaixo:

\begin{tabular}{ccccc}
\hline \hline Parâmetro & Estimativa & Erro Padrão & Estatística de Wald & P-Value \\
\hline \hline$\widehat{\alpha}$ & -8.335 & 0.4384 & 19.01 & $<0.01$ \\
$\widehat{\beta}$ & 0.0001494 & 0.000022 & 6.866 & $<0.01$ \\
\hline \hline
\end{tabular}

Como o P-value do teste de Wald para a hipótese $H_{0}: \beta=0$ é $<0.01$, esta hipótese é rejeitada. Em termos práticos, isto significa que as falhas experimentadas pelo motor de propulsão do USS Halfbeak, apresentam um comportamento de tendência (crescente ou decrescente) em função do tempo, ou seja, se comporta como um processo de Poison não-homogêneo.

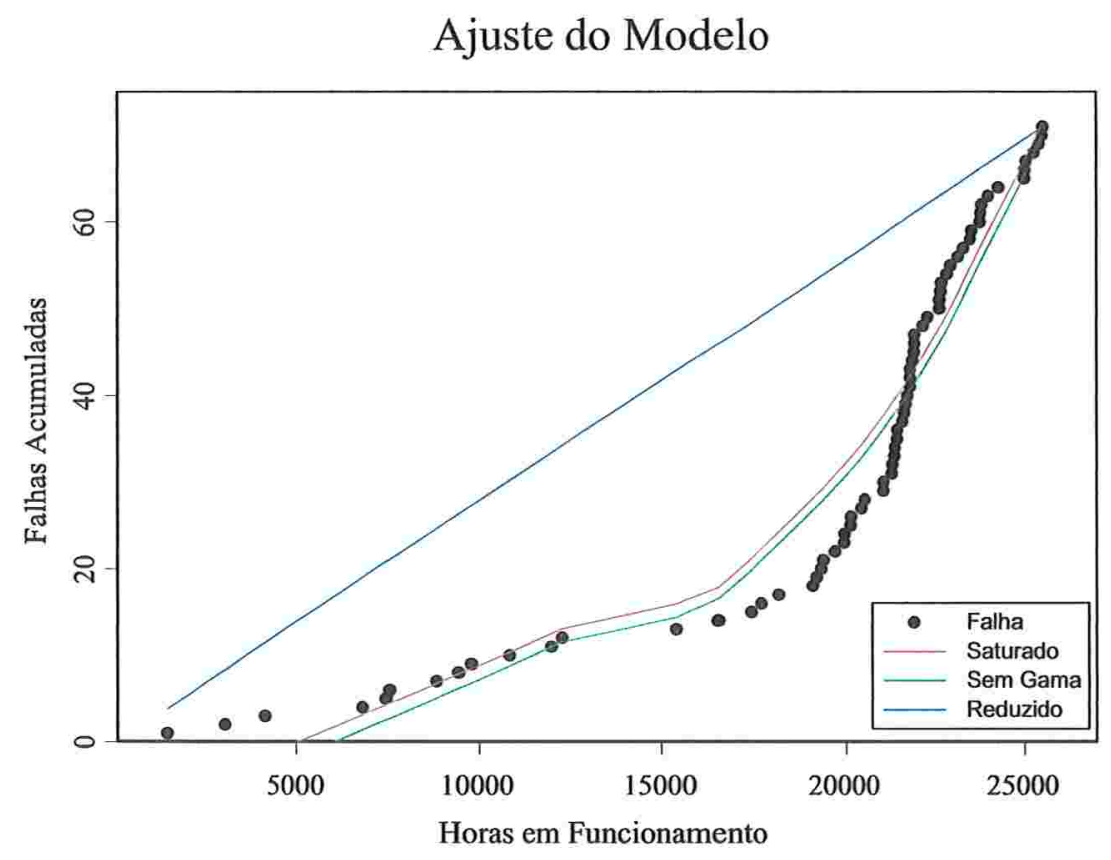

Figura 3.4: Comparação de Ajustes - USS Halfbeak 


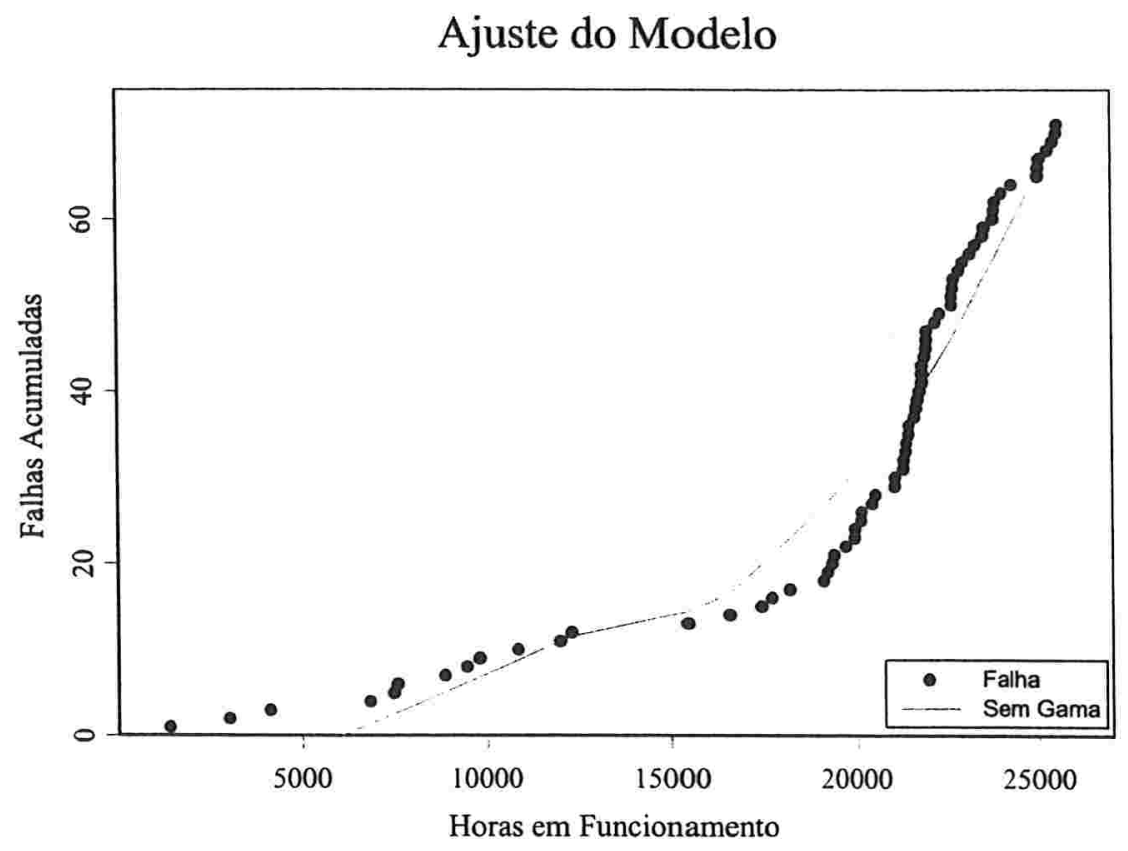

Figura 3.j: Modelo Ajustado - USS Halfbeak

Para verificar a qualidade do ajuste do modelo, iremos fazer uma análise dos resíduos. Utilizaremos como ferramenta um gráfico quantil-quantil dos resíduos generalizados.

De fato, o QQ-Plot da Figura 3.5 indica que os resíduos seguem uma distribuição exponencial padrão. Consequentemente, podemos considerar que o modelo reduzido está bem ajustado.

\subsection{Estudo de Caso III}

Neste estudo de caso vamos utilizar dados de falhas de quatro sistemas idênticos. Estes dados foram extraídos de [28].

Como modelo de base para análise, utilizaremos o modelo de processo pontual com função intensidade completa dada por: 


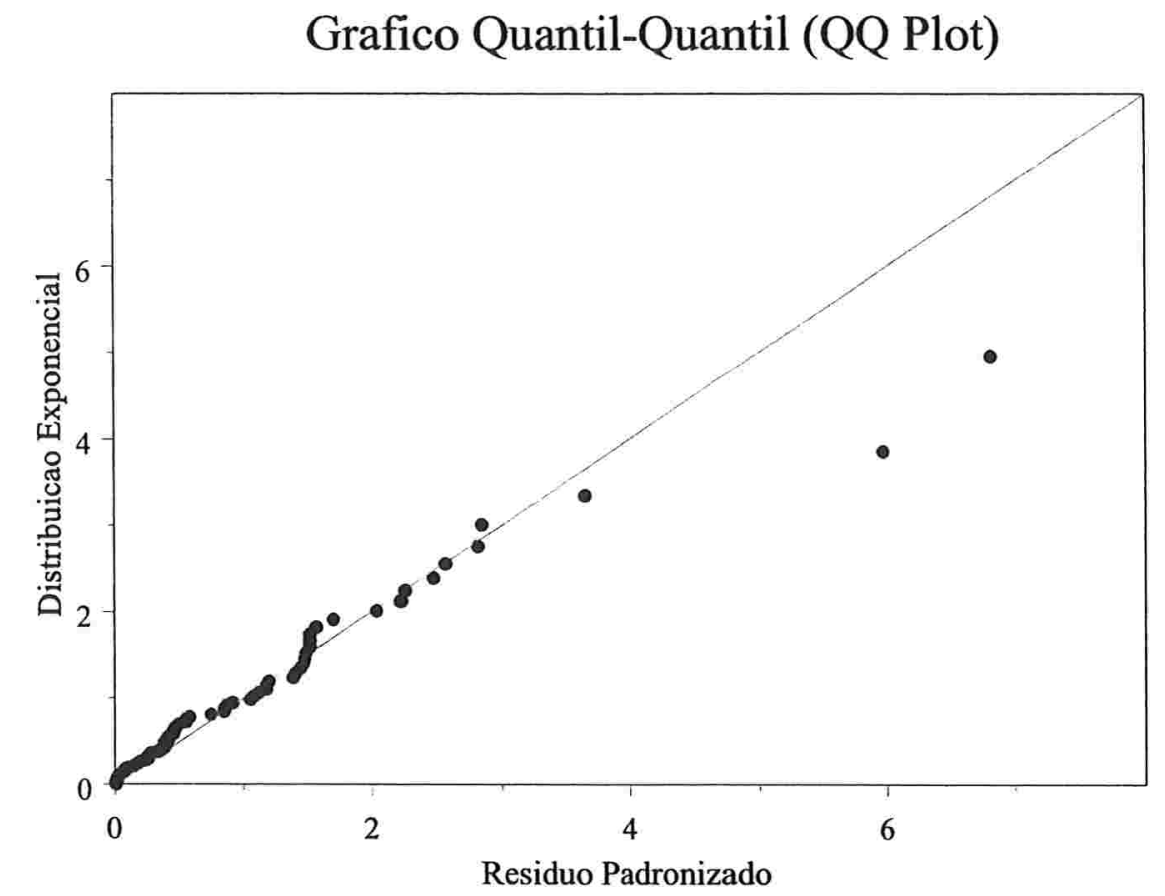

Figura 3.6: QQ Plot dos Resíduos - USS Halfbeak

$$
\lambda\left(t \mid N_{u}: u<t\right)=\exp \left\{\Theta^{T} \mathbf{Z}\left(t \mid N_{u}: u<t\right)\right\}
$$

com

$$
\Theta=\left(\begin{array}{c}
\alpha \\
\beta \\
\gamma
\end{array}\right), \mathbf{Z}(t)=\left(\begin{array}{c}
1 \\
t \\
t_{N_{t-}}
\end{array}\right)
$$

em que $t_{N_{t-}}$ é o tempo decorrido desde a última falha do sistema.

Os tempos de falhas utilizados na análise encontram-se na tabela abaixo: 


\begin{tabular}{cccccccccccc}
\hline \hline Sistema & Falhas & \multicolumn{10}{c}{ Tempos de Falhas } \\
\hline \hline 1 & 3 & 69 & 112 & 338 & & & & & & \\
2 & 7 & 14 & 26 & 67 & 90 & 213 & 244 & 301 & & \\
3 & 7 & 22 & 37 & 104 & 118 & 233 & 502 & 622 & & \\
4 & 9 & 5 & 41 & 57 & 105 & 240 & 341 & 503 & 655 & 701 \\
\hline \hline
\end{tabular}

Podemos também observar o fluxo de falhas experimentadas em cada um dos sistemas através do Figura 3.7.

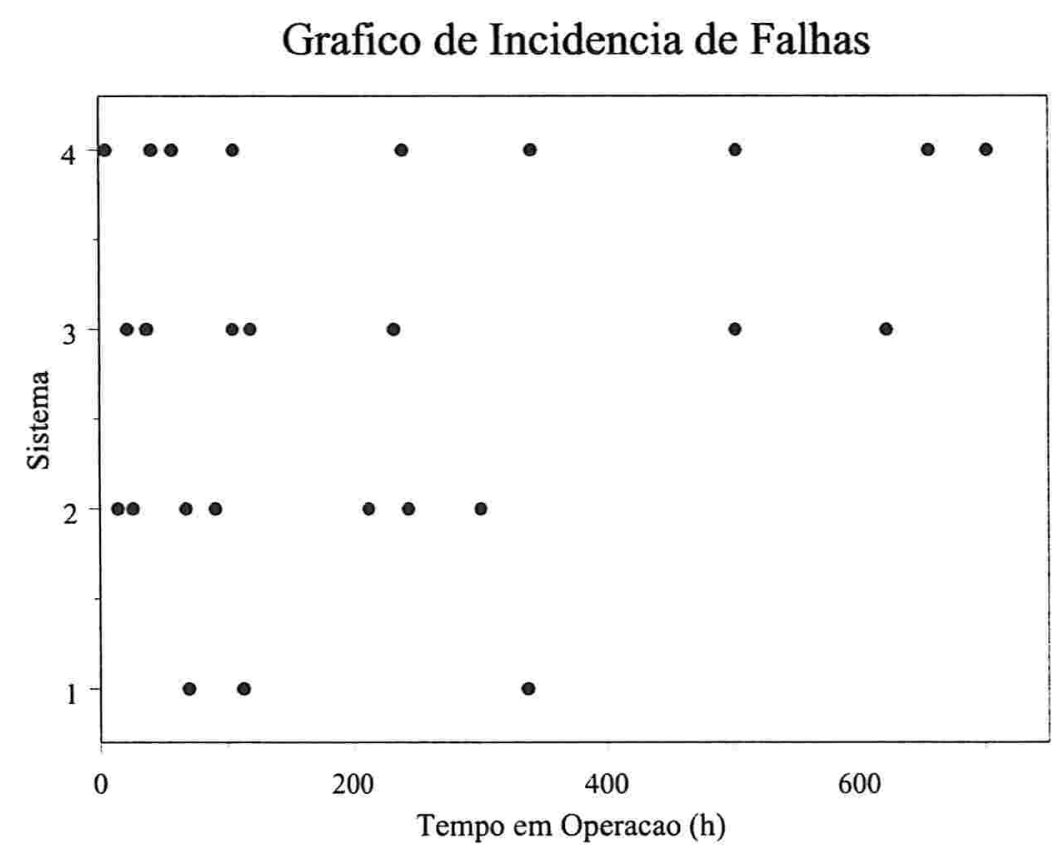

Figura 3.7: Incidência de Falhas por Sistema

Com base nos tempos de falhas observados e no modelo para a função de intensidade completa de falha especificada, as estimativas obtidas para os parâmetros do modelo encontram-se abaixo: 


\begin{tabular}{ccccc}
\hline \hline Parâmetro & Estimativa & Erro Padrão & Estatística de Wald & P-Value \\
\hline \hline$\widehat{\alpha}$ & -4.014 & 0.3441 & 136.10 & $<0.001$ \\
$\widehat{\beta}$ & -0.0031 & 0.0016 & 3.68 & 0.055 \\
$\widehat{\gamma}$ & 0.0063 & 0.0039 & 2.63 & 0.105 \\
\hline \hline
\end{tabular}

Podemos verificar, através do P-value do teste de Wald, que os efeitos de tendência e de renovação não são significantes ao nível de $5 \%$. Assim, não rejeitamos as hipóteses de que $H_{0}: \gamma=0$ e $H_{0}: \beta=0$.Isto posto, iremos calcular as estimativas do modelo levando este fato em consideração. As novas estimativas, encontram-se na tabela abaixo:

\begin{tabular}{ccccc}
\hline \hline Parâmetro & Estimativa & Erro Padrão & Estatística de Wald & P-Value \\
\hline \hline$\widehat{\alpha}$ & -4.324 & 0.1961 & 486 & $<0.001$ \\
\hline \hline
\end{tabular}

O gráfico com o ajuste do modelo para cada um dos sitemas modelado encontra-se na Figura 3.8 abaixo.

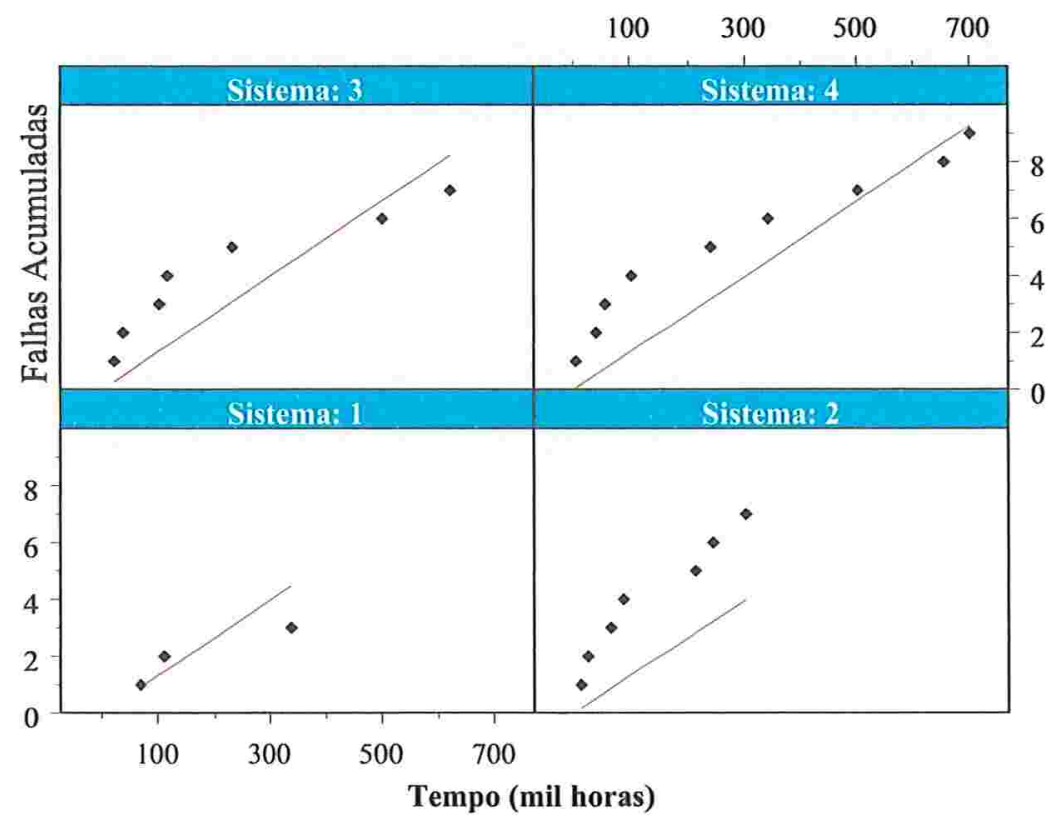

Figura 3.8: Modelo Ajustado - Sistemas 
Para verificar a qualidade do ajuste do modelo, iremos fazer uma análise dos resíduos. Utilizaremos como ferramenta um gráfico quantil-quantil dos resíduos generalizados.

\section{Grafico Quantil-Quantil (QQPlot)}

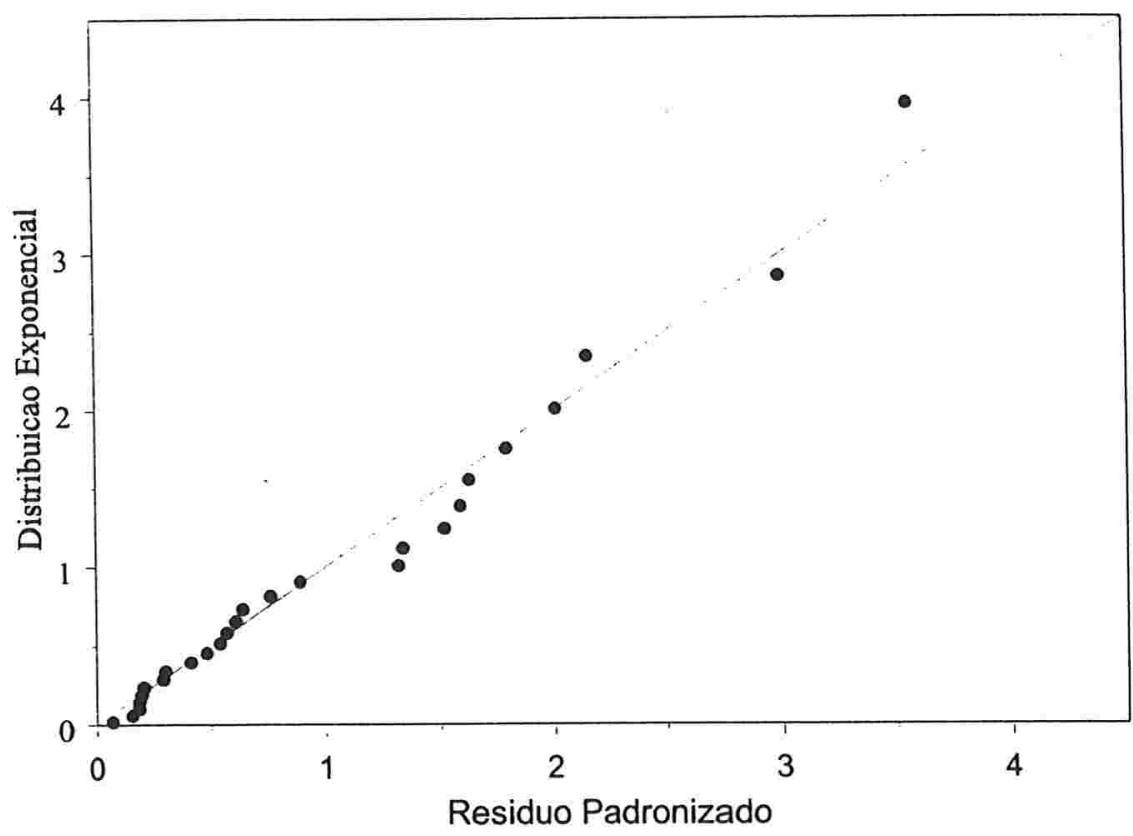

Figura 3.9: QQ Plot dos Resíduos - Sistemas

De fato, o QQ-Plot da Figura 3.9 indica que os resíduos seguem uma distribuição exponencial padrão. Consequentemente, podemos considerar que o modelo reduzido está bem ajustado.

Ressaltamos que a convergência dos métodos interativos para os cálculos das estimativas de máxima verossimilhança, quando trabalhamos com múltiplas unidades amostrais ou sistemas, pode não ocorrer. Um dos principais motivos para isto é a singularidade da matriz de fisher. Como poucos trabalhos na literatura especializada abordam esta questão deixamos seu estudo para trabalhos futuros. Como referência, a única que identificamos com um tratamento mais formal do tema é em [14]. 


\subsection{Considerações Finais}

Com os estudos e exemplos apresentados neste trabalho procurou-se demonstrar a extrema flexibilidade e facilidade de interpretação dos modelos mistos.

Para tanto, foi desenvolvido um software para análise estatística de dados de falha, com base nessa família de modelos para processos pontuais. Nas aplicações, entretanto, os vetores de funções intensidade completas ficaram restritos a componentes comumente encontrados na literatura especializada existente. Com isto, foi possível comparar os resultados obtidos por outros especialistas com aqueles resultantes da aplicação do software. Como foi visto, o aplicativo desenvolvido produziu resultados tão bons ou melhores do que os encontrados na literatura disponível.

A principal contribuição neste trabalho foi de natureza numérica, na obtenção dos estimadores de máxima verossimilhança dos parâmetros do modelo, para dados provenientes de múltiplos sistemas. Com o método proposto o algoritmo de aproximação tornou-se mais estável, convergindo para a solução com um número significativamente menor de iterações. Nestes casos, recomendamos ainda que os gráficos Quantil-Quantil dos resíduos generalizados sejam elaborados separadamente para cada uma das unidades amostrais. Isto tem como objetivo verificar possíveis fragilidades do modelo para cada unidade amostral. Este procedimento poderá indicar a necessidade de se ajustar um modelo específico para cada unidade amostral em questão, ou ajustar modelos específicos distintos para grupos de unidades amostrais.

Problemas desse tipo podem decorrer de fatores externos diferentes atuando sobre grupos de unidades amostrais. Note que apesar das unidades amostrais serem idênticas, elas podem estar sujeitas a níveis de estresse que impactam de forma profundamente diferente a modelagem e ajuste do modelo. Portanto, sugerimos como tópico futuro de pesquisa a incorporação de covariadas na modelagem das funções intensidade completas. Assim, será possível levar em considerações os distintos níveis de exposição ao estresse sofridos pelas unidades amostrais e, consequentemente, obter modelos melhores ajustados. 

Apêndice 


\section{Programa Fonte}

Todas as análises e gráficos apresentados nesta dissertação foram implementados através da linguagem S do software S-Plus, versão 6.2. Desta forma, deixamos alguns dos programas fontes utilizados como exemplo e também para um melhor entendimento da metodologia. Ressaltamos que, com base nos modelos descritos neste trabalho, uma gama de outros modelos podem ser implementados utilizando as funções de interesse.

O programa fonte anexo, foi desenvolvido para analizar a confiabilidade de uma única unidade amostral (sistema) com uma função intensidade completa dada por:

$$
\lambda\left(t \mid N_{u}: u<t\right)=\exp \left\{\Theta^{T} \mathbf{Z}\left(t \mid N_{u}: u<t\right)\right\}
$$

com

$$
\Theta=\left(\begin{array}{c}
\alpha \\
\beta \\
\gamma
\end{array}\right), \mathbf{Z}(t)=\left(\begin{array}{c}
1 \\
t \\
t-t_{N_{t-}}
\end{array}\right)
$$

Onde $t_{N_{t-}}$ é o tempo da última falha antes do instante $t$.

Ou seja, de uma função mista com uma componente log-linear e outra de renovação simples. Para ela o programa fonte escrito S-Plus que possibilita os cálculos das estimativas dos parâmetros e respectivos testes de significância, encontra-se abaixo:

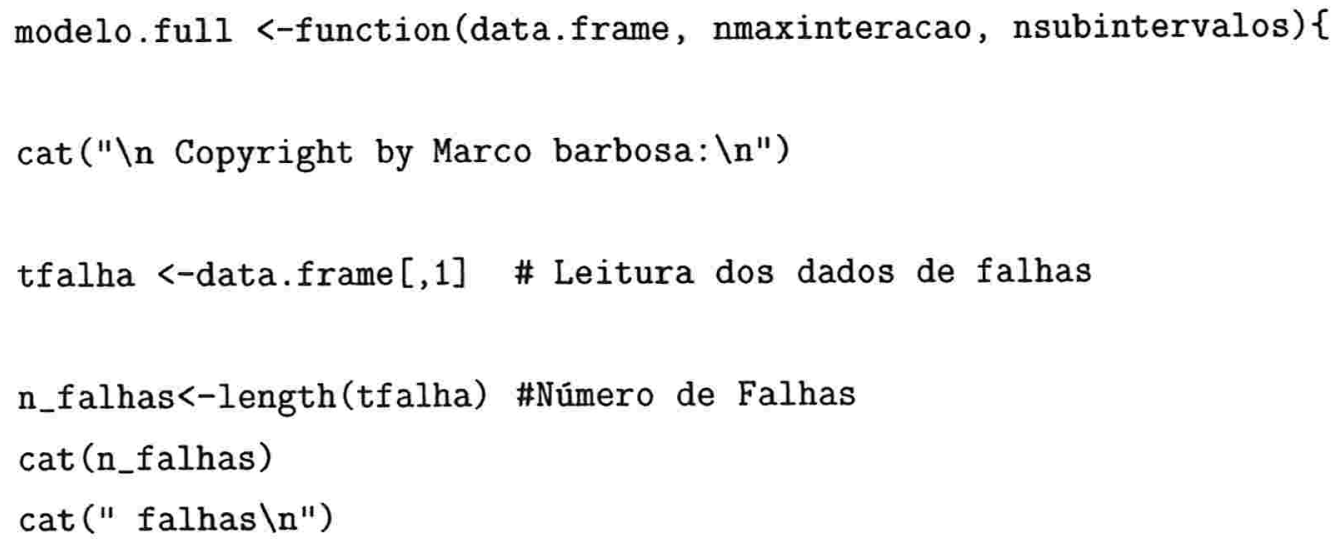




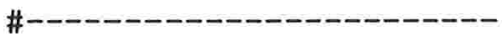

\# Definição das Variáveis

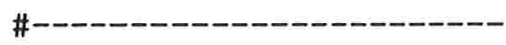

\# Número de falhas experimentadas pelo sistema

tamanho=n_falhas

\# Função Score para o parâmetro alfa

$\mathrm{Ua}<-1$ : tamanho

\# Função Score para o parâmetro beta

$\mathrm{Ub}<-1$ : tamanho

\# Função Score para o parâmetro gama

$\mathrm{Ug}<-1$ : tamanho

\#posição $[1,1]$ da matriz de informação, representando a o \# valor da segunda derivada da verossimilhança em

\#relação a alfa

Iaa<- 1: tamanho

\#posição $[1,2]$ da matriz de informação representando \# a derivada de Ua em relação a beta

Iab<- 1: tamanho

\#posição [1,3] da matriz de informação representando \# a derivada de Ua em relação a gama

Iag<- 1: tamanho

\#posição [2,2] da matriz de informação representando \# a segunda derivada da verossimilhança em relação a beta Ibb<- 1: tamanho 
\#posição [2,3] da matriz de informação que representa \# a derivada de Ub em relação a gama

Ibg<- 1: tamanho

\#posição [3,3] da matriz de informação que representando \# a segunda derivada da verossimilhança em relação a gama Igg<- 1: tamanho

\#vetor que armazena, para cada interação o valor da função \# de verossimilhança calculada para os respectivos valores \# dos parâmetros veross<- 1 : tamanho

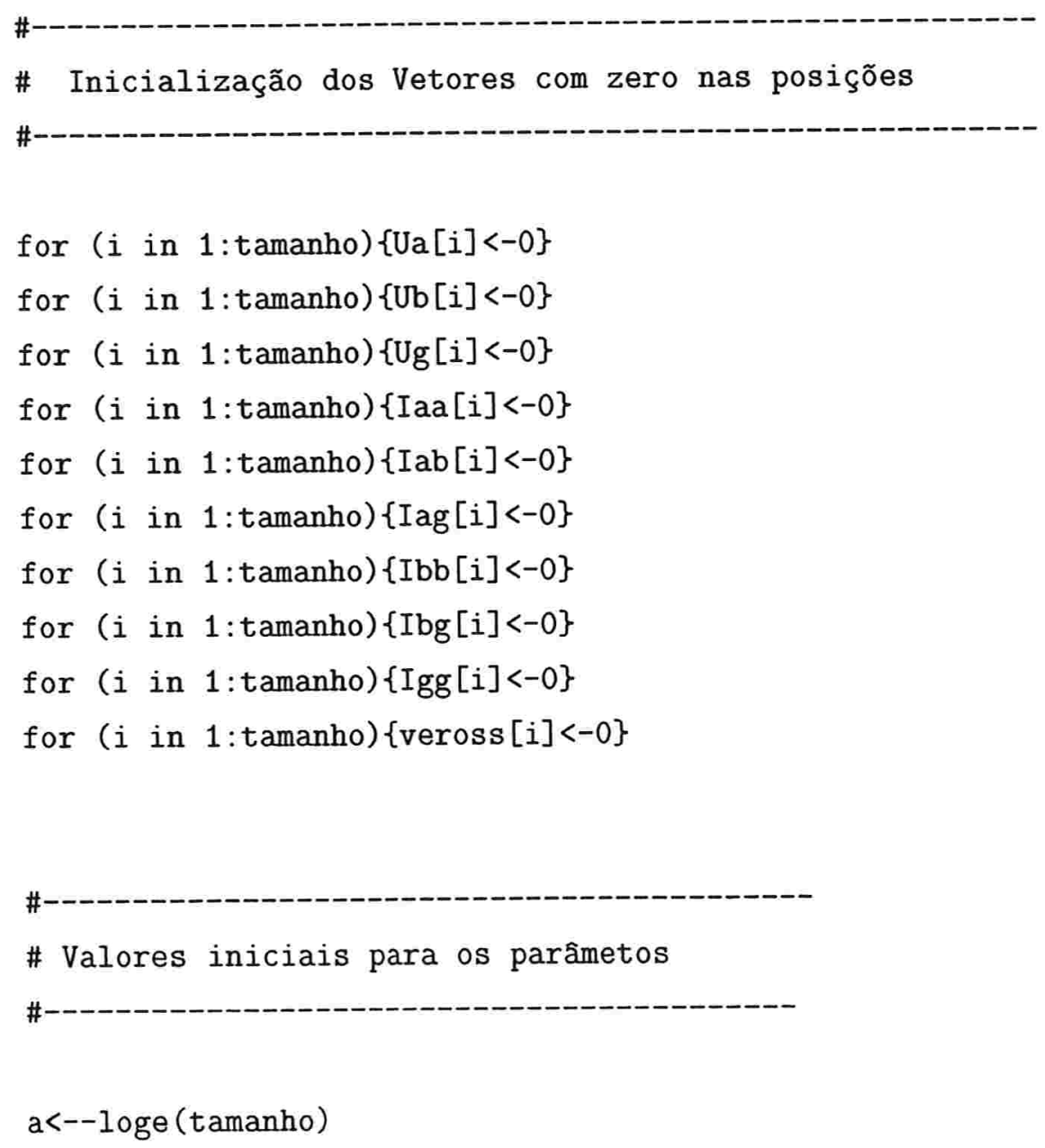


$\mathrm{b}<-0$

$\mathrm{g}<--0$

$\#-$

\#monta o vetor de parâmetros

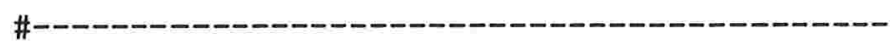

Theta1<-matrix $(c(a, b, g)$, nrow $=3)$

\#--

\# Processo Interativo

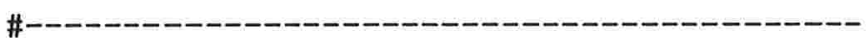

for ( $\mathrm{k}$ in 1: nmaxinteracao) \{

\# Em cada interação os valores das variáveis são

\# zerados

peso $<-0$

SUa $<-0$

$\mathrm{SUb}<-0$

SUg $<-0$

SIaa $<-0$

$\mathrm{SIab}<-0$

SIag $<-0$

SIbb $<-0$

SIbg $<-0$

SIgg $<-0$

tinicial $<-0$

tf inal<-0

delta<-0

vero<-0 


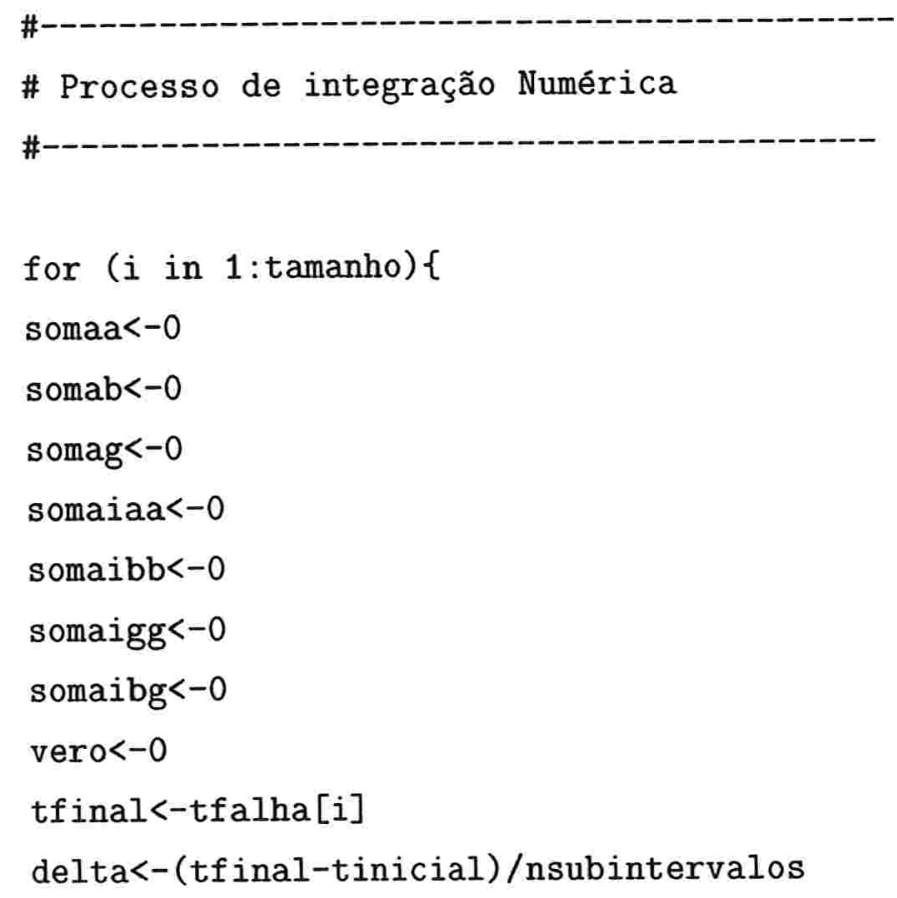




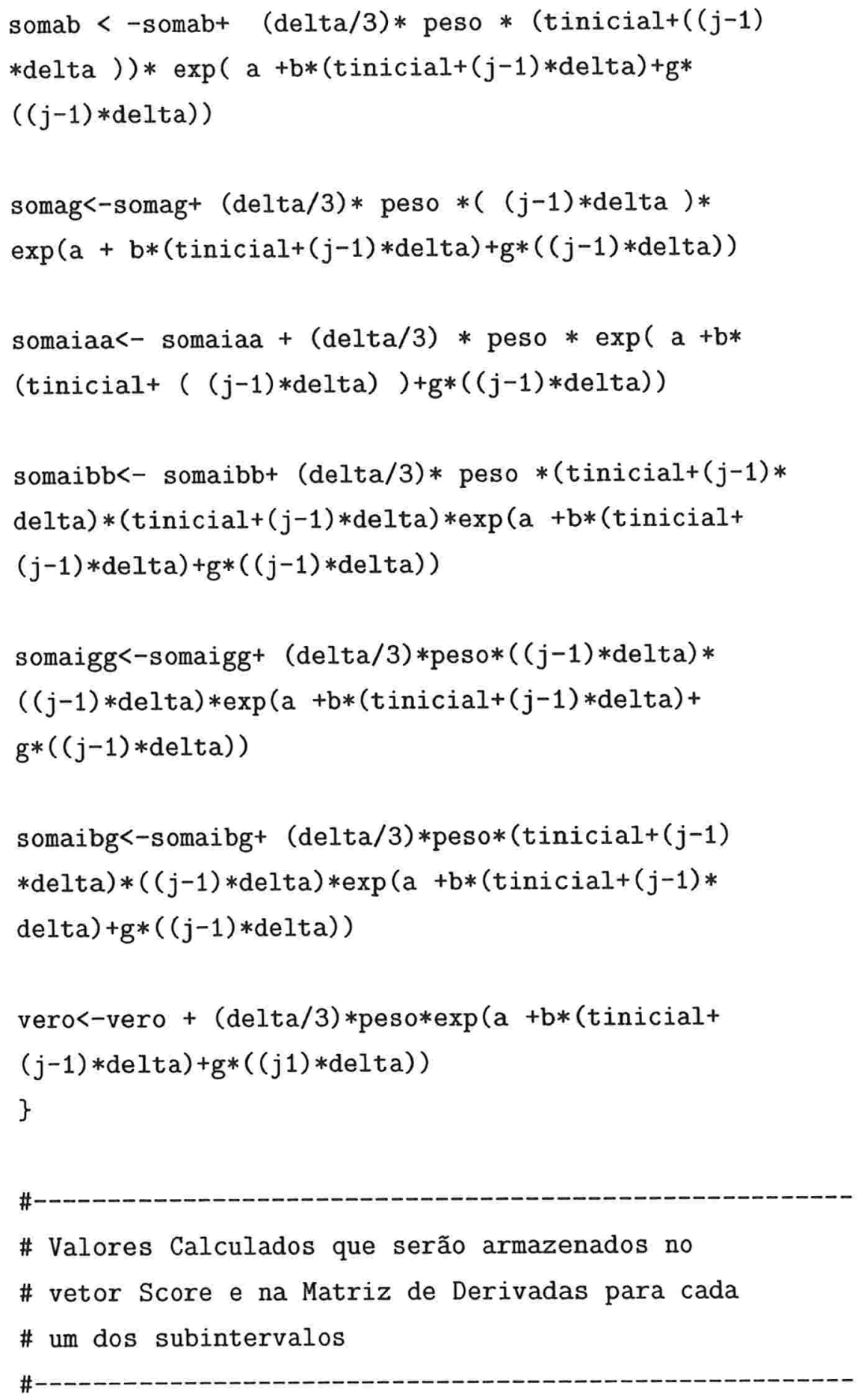




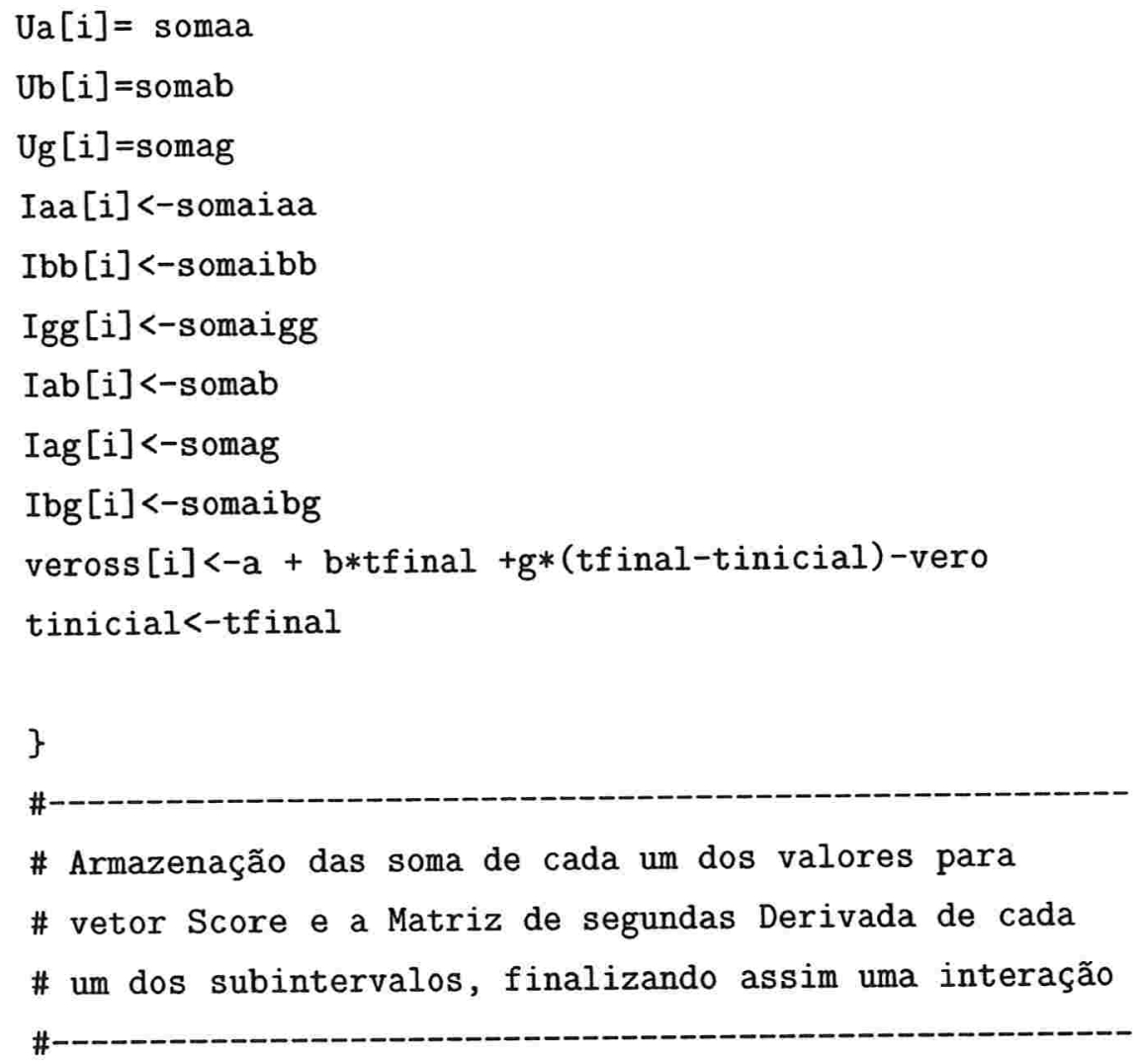




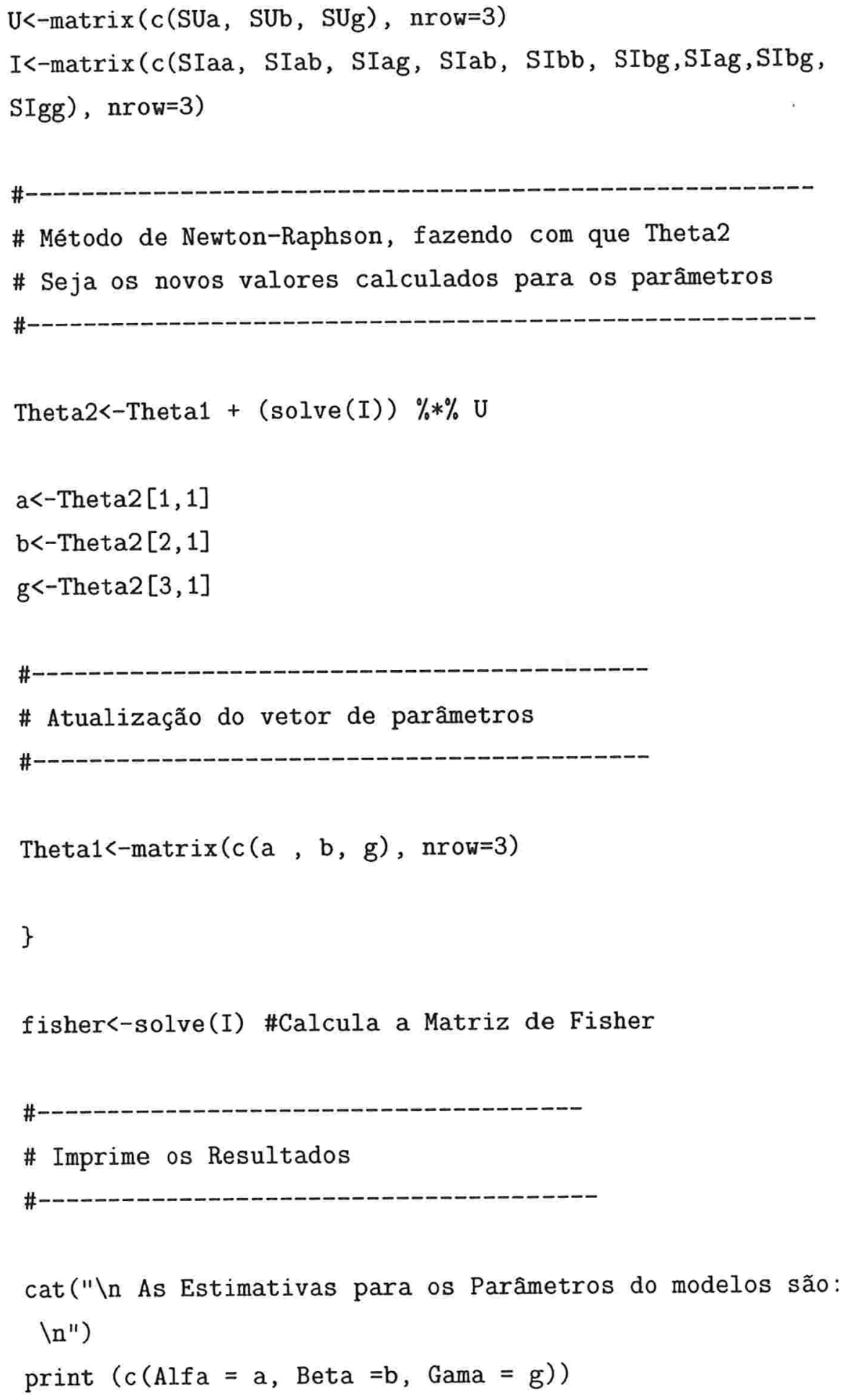




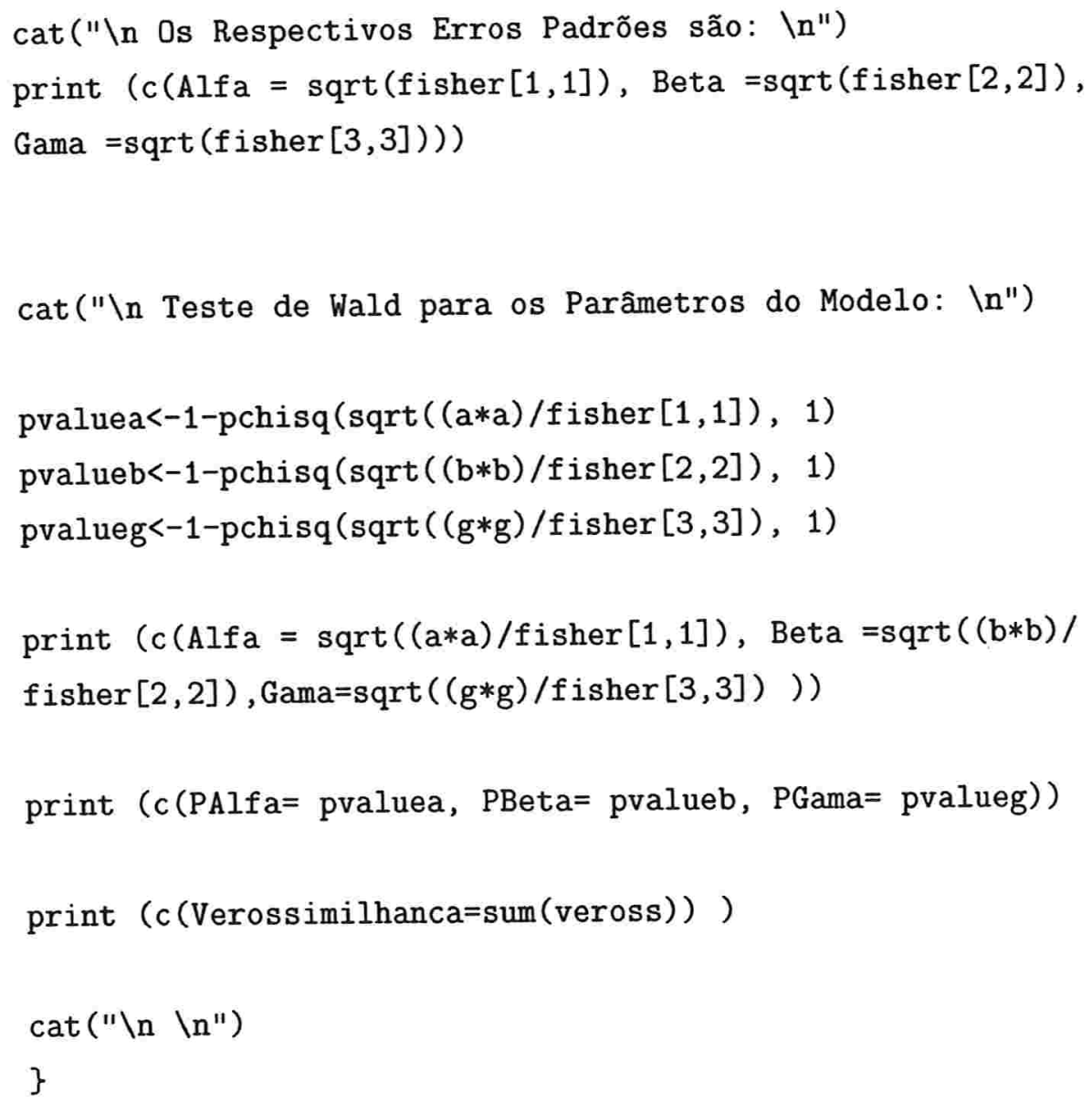

Os programas referentes a análise de sistemas múltiplos são mais complexos e uma boa inicialização do vetor de parâmetros é necessária para que haja a convergência do método iterativo. 


\section{Referências Bibliográficas}

[1] Andersen, Per Kragh, Borgan, Ornulf, Gill, Richard D. e Keidding, Niels(1992). Statistical Models Based on Counting Process. Springer, New York.

[2] Asher, Harold e Feingold, Harry (1984). Repairable Systems Reliability: Modeling, Inference, Misconceptions and their Causes. Marcel Dekker, Inc., New York.

[3] Aven, Terje e Jensen, Uwe (1991). Stochstic Models in Reliability. Springer, New York.

[4] Barlow, Richard E.(1998). Engineering Reliability. ASA-SIAM, Berkeley.

[5] Bartlett, M. S.(1963). The Spectral Analysis Of Point Process. Jornal Of Royal Society Of London, Series B, Vol. 25, pp.264-296.

[6] Berman, M.(1981). Inhomogeneos And Modulated Gamma Processes. Biometrika, Vol. 68, pp. 143-152.

[7] Borges, W. S., Block, H. W. e Savits, T.H.(1985). Age-Dependent Minimal Repair. Journal of Applied Probability, Vol 22, pp. 370-375.

[8] Casella, George e Berger, Roger L.(1990). Statistical Inference. Duxbury Press, Belmont.

[9] Çinlar, Erhan(1975). Introduction to Stochastic Process. Prentice-Hall, New Jersey. 
[10] Cox, D. R. e Lewis, P. A. W.(1966). The Statistical Analysis Of Series Of Events. CHAPMAN \& HALL, London.

[11] Crowder, M. J., Kimber, A. C., Smith, R. L. e Sweeting, T. J.(2000). Statistical Analisys of Reliability Data. CHAPMAN \& HALL, London.

[12] Daley, D. J. e Vere-Jones, D.(2002). An Introduction to The Theory of Point Process : Elementary Theory and Methods. 2nd. edition, Springer, New York.

[13] Ebeling, Charles E.(1997). Reliability and Maintainability Engineering. McGRAW-HILL, San Francisco.

[14] Lindqvist, B.H., Elvebakk, G. e Heggland, K. (2003) The Trend-Renewal Process for Statistical Analysis of Reparaible Systens. Technometrics. Vol 45 (1), 31-44.

[15] Hoyland, Arnljot e Rausand, Marvin(2003). System Reliability Theory: Models and Statistical Methods. John Wiley, New York.

[16] Ireson, W. Grant, Coombs, Clyde F. e Moss, Richard Y.(1995). Handbook of Reliability Engineering and Management. 2nd. edition, McGRAWHILL, New York.

[17] Kumamoto, Hiromitsu e Henley, Ernest J.(1996). Probabilistic Risk Assessment and Management for Engineers and Scientists. 2nd. edition CHAPMAN \& HALL, London.

[18] Kalbfleish, John D., Prentice, Ross L.(1980). The Statistical Analysis of failure Time Data. John Wiley, New York.

[19] Kahle, Waltraud, Collani, Elart, Franz, Jürgen e Jensen, Uwe (1998). Advances in Stochastic Models for Reliability, Quality and Safety. Birkhäuser, Boston.

[20] Lawless, Jerald F. e Thiagarajah, K.(1996). A Point Process Model Incorporating Renewals and Time Trends, With Applications to Repairable Systems. Technometrics. Vol 38 (2), 131-138. 
[21] Lawless, Jerald F.(2004). Statistical Models and Methods for Lifetime Data. 2nd. edition, IEEE PRESS, New York.

[22] Lehmann, E. L.(1997). Testing Statistical Hypotheses. 2nd. edition, Springer, New York.

[23] Lehmann, E. L. e Casella, George(1998). Theory of Point Estimation. 2nd. edition, Springer, New York.

[24] Meeker, Willian Q., Escobar, Luis A.(1998). Statistical Methods for Reliability Data. John Wiley, New York.

[25] Nelson, Wayne(1982). Applied Life Data Analysis. John Wiley, New York.

[26] Nelson, Wayne(1990). Accelerate Testing: Statistical Models, Test Plans and Data Analysis. John Wiley, New York.

[27] Nelson, Wayne(2003). Recurrent Events Data Analysis for Product Repairs, Disease Recurrences, and Other Applications. ASA-SIAM, New York.

[28] Rigdon, Steven E. e Basu, Asit P.(2000). Statistical Methods for the Reliability of Repairable Systems. John Wiley, New York.

[29] Robert, Christian P. e Casella, Gorge(1999). Monte Carlo Statistical Methods. Springer, New York.

[30] Ross, Sheldon M.(1996). Stochastic Process. 2nd. edition, John Wiley, New York.

[31] Ross, Sheldon M.(2002). Simulation. 3th. edition, Academic Press, San Diego.

[32] Ruggiero, Márcia A. G. e Lopes, Vera L. R. (1988). Cálculo Numérico: Aspectos Teóricos e Computacionais. McGRAW-HILL, São Paulo.

[33] Sen, A e Bhattacharyya, G. K. (1993). A Piecewise Exponential Model For Reliability Growth And Associated Inferences. Advances in Reliability, pp. 35う-370. 
[34] Serfling, Robert J.(2002). Approximation Theorems of Mathematical Statistics. John Wiley, New York.

[35] Tobias, Paul A. e Trindade, David C.(1998). Applied Reliability. 2nd. edition CHAPMAN \& HALL, London.

[36] Venables, W. N. e Ripley, B. D.(2002). Modern Applied Statistics with $S$. 4th. edition, Springer, New York. 